\title{
Single base substitutions in the CHM promoter as a cause of choroideremia
}

\begin{tabular}{|r|l|}
\hline Journal: & Human Mutation \\
\hline Manuscript ID & humu-2016-0385.R2 \\
\hline Wiley - Manuscript type: & Research Article \\
\hline Date Submitted by the Author: & n/a \\
\hline Complete List of Authors: & $\begin{array}{l}\text { Radziwon, Alina; University of Alberta, Ophthalmology } \\
\text { Arno, Gavin; University College London, Institute of Ophthalmology; } \\
\text { Moorfields Eye Hospital } \\
\text { Wheaton, Dianna; Retina Foundation of the Southwest, } \\
\text { McDonagh, Ellen; Queen Mary University of London, Genomics England } \\
\text { Baple, Emma; Queen Mary University of London, Genomics England; } \\
\text { University of Exeter, RILD Wellcome Wolfson Centre } \\
\text { Webb, Kaylie; Retina Foundation of the Southwest } \\
\text { Birch, David; Retina Foundation of the Southwest } \\
\text { Webster, Andrew; University College London, Institute of Ophthalmology; } \\
\text { Moorfields Eye Hospital } \\
\text { MacDonald, Ian; University of Alberta, Ophthalmology }\end{array}$ \\
\hline Key Words: & \begin{tabular}{l} 
choroideremia, CHM, REP-1, ZNF143, promoter, THAP11 \\
\hline
\end{tabular} \\
\hline
\end{tabular}

\section{SCHOLARONE ${ }^{\text {m }}$}

Manuscripts 
1 Single base substitutions in the $C H M$ promoter as a cause of choroideremia

2

3 Alina Radziwon $^{1 *}$, Gavin Arno ${ }^{2,3}$, Dianna Wheaton ${ }^{4}$, Ellen M. McDonagh ${ }^{5}$, Emma L. Baple ${ }^{5,6}$,

4 Kaylie Webb-Jones ${ }^{4}$, David Birch ${ }^{4}$, Andrew R. Webster ${ }^{2,3}$, Ian M. MacDonald ${ }^{1}$

5

$6 \quad{ }^{1}$ Department of Ophthalmology and Visual Sciences, University of Alberta, Edmonton, Canada

$7 \quad{ }^{2}$ UCL Institute of Ophthalmology, University College London, London EC1V 9EL, UK

$8 \quad{ }^{3}$ Moorfields Eye Hospital, London EC1V 2PD, UK

$9{ }^{4}$ Retina Foundation of the Southwest, Dallas, Texas, United States of America

$10{ }^{5}$ Genomics England, Queen Mary University of London, Charterhouse Square, London, EC1M

11 6BQ, UK

$12{ }^{6}$ Medical Research (Level 4), University of Exeter Medical School, RILD Wellcome Wolfson

13 Centre, Royal Devon and Exeter NHS Foundation Trust, Barrack Road, Exeter, EX2 5DW, UK

14

15 *Correspondence: Alina Radziwon, Department of Ophthalmology and Visual Sciences, 7-030

16 Katz Building, University of Alberta, Edmonton, Alberta, Canada, T6G 2E1. Phone: 1-780-492-

17 1141; e-mail: alinar@ualberta.ca

18

19 Presented at: The Association for Research in Vision and Ophthalmology Annual Meeting, May 20 2016, Seattle, WA, USA.

21 
Promoter mutation causing choroideremia

22 Financial Support: IM: Canadian Institutes of Health Research, Emerging Team Grant: 119190,

23 Foundation Fighting Blindness, Canada, Choroideremia Research Foundation Canada, Inc., and

24 Alberta Innovates-Health Solutions 201201139

25 DB: National Eye Institute grant EY-09076, Foundation Fighting Blindness

26 GA, AW: The National Institute for Health Research (NIHR) Biomedical Research Centre

27 (BRC) at Moorfields Eye Hospital, and the UCL Institute of Ophthalmology. This research was

28 made possible through access to the data and findings generated by the 100,000 Genomes

29 Project. The 100,000 Genomes Project is managed by Genomics England Limited (a wholly

30 owned company of the Department of Health). The 100,000 Genomes Project is funded by the

31 NIHR and NHS England. The Wellcome Trust, Cancer Research UK and the Medical Research

32 Council have also funded research infrastructure. 
Promoter mutation causing choroideremia

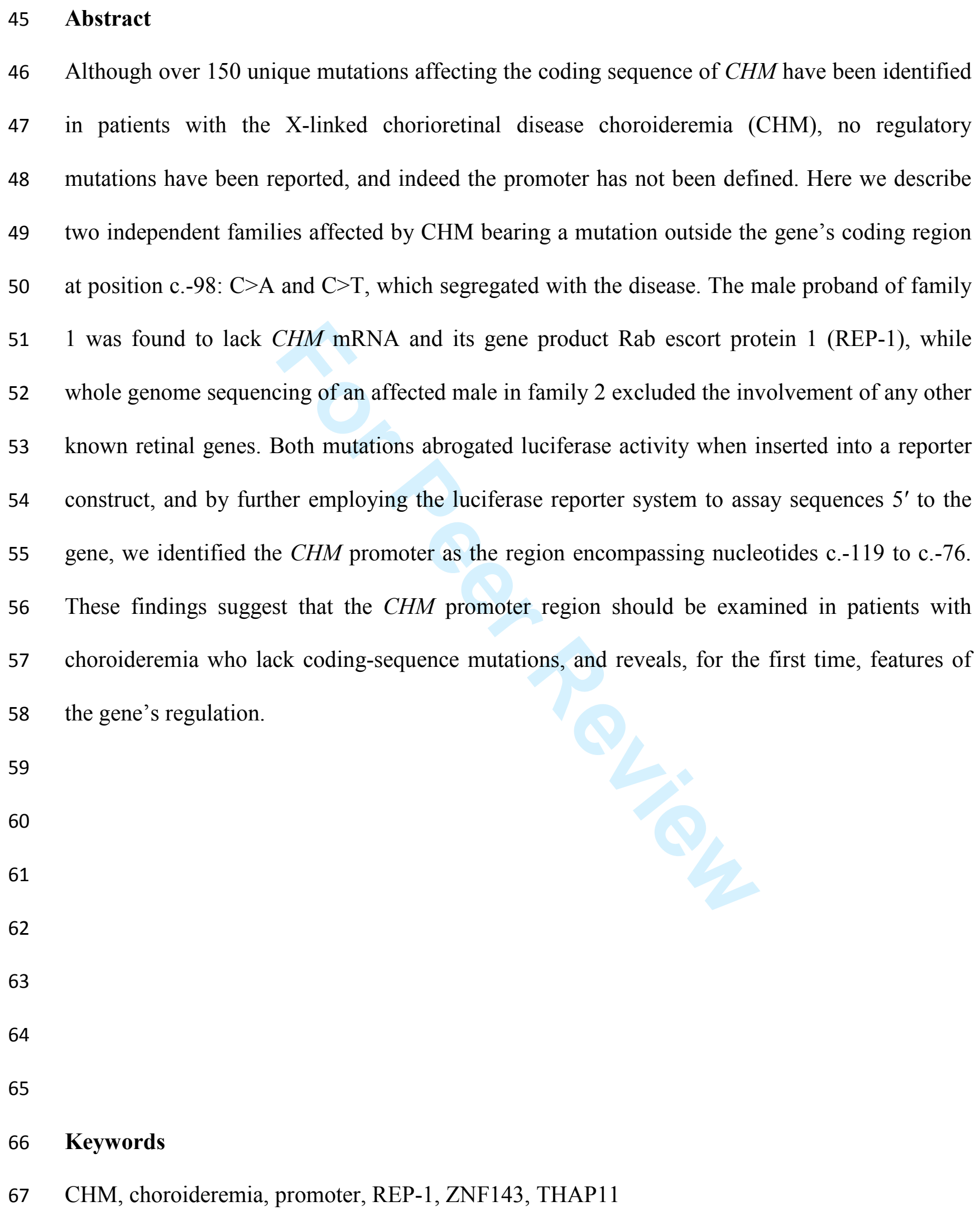


Promoter mutation causing choroideremia

\section{Introduction}

Choroideremia (CHM; MIM\# 303100) is an X-linked, recessively inherited chorioretinal dystrophy with an incidence of 1/50,000. Progressive degeneration of photoreceptors, retinal pigment epithelium (RPE), and the choroid causes affected hemizygous males to develop night blindness in the first or second decade of life, followed by a decrease in peripheral visual fields and an eventual loss of central visual acuity in advanced stages of the disease (Coussa and Traboulsi, 2012). Carrier females, while usually asymptomatic, may exhibit signs of retinal degeneration upon fundoscopic examination, and more rarely have reduced dark adaptation and peripheral vision (Karna, 1986; Roberts et al., 2002).

To date, CHM has only been linked to mutations within the $C H M$ gene, coding for REP-1 (Cremers et al., 1990). The protein serves as a molecular chaperone for small GTPases from the Rab family, presenting them to Rab geranylgeranyl transferase which modifies them by the covalent attachment of a lipid moiety. The lipid modification, known as prenylation, of target Rab proteins is essential for intracellular vesicular transport (Seabra et al., 1992; Seabra et al., 1993). A homologue, REP-2, encoded by $C H M L$ or choroideremia-like, functions similarly and appears to compensate for the absence of REP-1 in all tissues except the eye (Cremers et al., 1994). CHM spans over $150 \mathrm{~kb}$ of Xq21.2 and contains 15 exons (van Bokhoven et al., 1994). A 30 base pair 5'untranslated region, found on exon 1, precedes an open reading frame encoding the 653 amino acid REP-1 protein. At least 163 unique pathogenic mutations have been reported

87 in the LOVD Retinal and Hearing Impairment Genetic Mutation Database 8 [https://grenada.lumc.nl/LOVD2/Usher_montpellier/home.php; accessed Feb 2017] (Fokkema et al., 2011). The mutation spectrum includes transitions and transversions leading to protein truncation, splice defects, indels and large deletions ranging from a single exon to the full gene. 
91 Missense mutations predicted to alter protein structure or impair function (Sergeev et al., 2009;

92 Esposito et al., 2011), transposon insertions (Van Den Hurk et al., 2003), partial gene

93 duplications (Chi et al., 2013; Simunovic et al., 2016) and other variations are infrequently

94 found, but taken collectively, almost all known pathogenic variants in the $C H M$ gene have been 95 loss of function mutations that abolish functional REP-1 (McTaggart et al., 2002; Simunovic et 96 al., 2016). Notably there is no apparent correlation between genotype and phenotype, with the 97 age at onset of symptoms, visual acuity and visual fields being unrelated to mutation type 98 (Freund et al., 2016; Simunovic et al., 2016).

99 The promoter driving expression of $C H M$ has been heretofore unidentified. Analyses of 100 promoter mutations causing inherited diseases can be useful in identifying transcription factors 101 involved in the regulation and expression of a gene of interest, as the activity of RNA 102 polymerase II is mediated by the recruitment of general and sequence specific transcription 103 factors to cis-acting regulatory sequences. The core promoter directing basal level transcription 104 is generally found between nucleotides -40 and +50 of the transcription start site (TSS) (de 105 Vooght et al., 2009), while proximal promoter elements typically reside within 1 kilo bases (kb), 106 with additional enhancers, repressors, insulators acting even over distances of mega bases 107 (Maston et al., 2006). The investigation of regulatory elements in the context of human disease is 108 complicated by the fact that in contrast to loss of function mutations, regulatory defects may 109 produce small quantitative changes that are difficult to detect. Mutations in cis-acting regulatory 110 sequences are a significant cause of human disease, and according to statistics compiled by the 111 Human Gene Mutation Database [http://www.hgmd.cf.ac.uk ; accessed May 2016] 112 approximately $2 \%$ of disease causing point mutations are in non-coding regions of the genome 113 (Stenson et al., 2014). Though promoter defects can cause functionally important consequences 
114 for gene expression, their analysis is often not a regular part of DNA diagnostics as the 115 investigation can be complex, laborious and difficult to perform.

116 In this study, we report the first known regulatory mutations causing choroideremia. The absence 117 of a genetic defect in a CHM family prompted us to explore the upstream non-coding region, 118 revealing the novel promoter variant c.-98C $>$ A. Subsequently, whole genome sequencing in an 119 unrelated family with $\mathrm{CHM}$ identified a second variant of the same residue, c.-98C $>\mathrm{T}$. We 120 demonstrate the mutations' effects on transcription using a luciferase assay, and employ the same 121 system to further characterize the boundary of the CHM promoter that spans the location. The 122 results suggest a disruption of a transcription factor binding site and impaired transactivation of 123 the CHM promoter by the factor(s). Together, these studies further our understanding of 124 regulation and expression of $C H M$ and present a possible explanation for cases of unexplained 125 choroideremia where no causative mutation is found within the gene.

127 Materials and Methods

128

129 Clinical Examination and Study Subjects

130 This study was approved by the institutions' respective ethics review boards. All procedures 131 adhered to the tenets of the Declaration of Helsinki. Before participation, the purpose and risks 132 of the study were explained, and informed consent was obtained. Blood samples were drawn, 133 and a detailed pedigree and history was recorded.

134 We studied 4 males affected by $\mathrm{CHM}$ and 1 carrier female from two unrelated families. 135 Individuals were examined by fundoscopy and functional ophthalmologic methods including 136 some or all of: visual acuity testing, central visual fields, full field electroretinography, optical 
137 coherence tomography and fundus autofluorescence imaging. Choroideremia was diagnosed by 138 senior clinicians with expertise in inherited retinal disease based on clinical findings alone. 139 Affected males had a history of night vision loss and fundus appearance of peripheral retinopathy 140 with broad areas of RPE and choroidal atrophy.

\section{CHM Sanger Sequencing}

143 DNA was extracted from lymphocytes using conventional methodologies. Proband 5116 from 144 family 1 (C127) was initially screened for $C H M$ mutations in the coding sequence and splice 145 sites by direct Sanger sequencing from PCR amplicons (Furgoch et al., 2014). Additionally, 1kb 146 of the 5'-flanking sequence was amplified with primers 5'-CAGGGAAGGCCCACTACTGC -3' 147 and 5'-CTTGTGGAAATGAGATCAAGTTAGG-3' and sequenced with the same primers. With 148 the exception of patient 111 and his parents, of family 2 who underwent whole genome 149 sequencing, the remaining study individuals were genotyped only at position c.-98 to confirm the 150 presence or absence of the respective variant. Annotation is in accordance with GenBank: 151 NM_000390.3, where +1 represents the start of translation.

\section{Cell Culture}

154 Leukocytes from patient 5116 were separated with Ficoll-Histopaque (Sigma-Aldrich, St. Louis, 155 MO, USA) from $10 \mathrm{~mL}$ of whole human blood collected in acid citrate dextrose (ACD-A) tubes 156 (BD, Franklin Lakes, NJ, USA). A lymphoblastoid cell line was established by Epstein-Barr 157 virus transformation within 48 hours of collection (Anderson and Gusella, 1984). Cells were 158 maintained in RPMI-1640 supplemented with $15 \%$ fetal calf serum and penicillin-streptomycin. 
Promoter mutation causing choroideremia

160

161

162

163

164

165

166

167

168

169

170 intron-spanning primers.

171 Protein was extracted from $2 \times 10^{6}$ lymphoblastoid cells and immunoblot analysis of REP-1 was 172 performed as previously described (Furgoch et al., 2014).
173

174

175

176

177

178 sequenced using an Illumina HiSeq X Ten, generating minimum coverage of $15 \mathrm{X}$ for $>97 \%$ of

179 the callable autosomal genome. Reads were aligned to build GRCh37 of the human genome 180 using the Isaac aligner (Illumina Inc). SNVs and indels were identified using Platypus v0.8.1 and 181 annotated using Cellbase (https://github.com/opencb/cellbase). Variant filtering was performed 182 using minor allele frequency (MAF) in publicly available and in-house datasets, predicted 
183 protein impact and familial segregation. Surviving variants were prioritized using a pre-specified 184 virtual gene panel from PanelApp (https://panelapp.extge.co.uk/crowdsourcing/PanelApp/ 185 Posterior segment abnormalities v1.7. Allelic state was required to match the curated mode of 186 inheritance for variants in panel genes.

\section{$188 \quad$ Promoter Analysis}

189 Construction of luciferase reporter plasmids

190 Primers were initially designed to amplify a 1063 bp fragment from upstream of the CHM 191 transcription start site (c.-1093_c.-31) from control and patient 5116's DNA. The c.-98C >T 192 mutation present in family 2 was generated by site directed mutagenesis from the control 193 plasmid. All sequences cloned contained only untranscribed DNA and did not include the 30 194 base pair 5'UTR directly upstream of the start codon. The fragment was cloned according to 195 standard protocols, with SacI and HindIII restriction sites added to primers allowing digest by 196 the enzymes (Thermo Scientific, Rockford, IL, USA). Ligation into the promoterless firefly 197 luciferase reporter vector, pGL3-Basic (Promega, Madison, WI, USA) generated constructs 198 pGL3-1063_-31 and pGL3-1063_-31 c.-98 C>A. Subsequently, a 4kb fragment, and a series of 199 nested deletions ranging from $2 \mathrm{~kb}$ to $44 \mathrm{bp}$ were cloned into pGL3-Basic with the same 200 restriction sites added and used for subsequent digest, to identify the minimal promoter necessary 201 and sufficient for transcription. pGL3-Basic lacks a eukaryotic promoter sequence upstream of 202 the reporter luciferase gene, and expression of luciferase in transfected cells depends on a 203 functional promoter sequence to be inserted upstream of the $l u c+$ gene. pGL3-Basic itself served 204 as a negative control. A schematic of all inserts assayed is provided in Figure 1A. All constructs 
Promoter mutation causing choroideremia

205 generated were Sanger sequenced to ensure fidelity. Primer sequences for the generation of 206 plasmid inserts are available upon request.

\section{Luciferase Assay}

208 HEK293T cells cultured with standard reagents and conditions were seeded in 24 well plates and 209 transfected at $70 \%$ confluency with polyethylenimine (PEI). Each well was transfected with a 210 total of 650ng of plasmid DNA, including 600ng of pGL3 construct and 50ng of the internal 211 control pRL-CMV, a cytomegalovirus promoter driven Renilla luciferase reporter vector. 212 Briefly, plasmid DNA was diluted into $25 \mu \mathrm{L}$ of PBS, and mixed by vortexing with $1 \mu \mathrm{L}$ of $2131 \mathrm{mg} / \mathrm{mL}$ branched PEI (Sigma-Aldrich, St. Louis, MO, USA) in $25 \mu \mathrm{L}$ PBS. After a 15 minute 214 incubation, $50 \mu \mathrm{L}$ of the reaction was added dropwise to each well. Forty-eight hours after 215 transfection, luciferase activity was assayed using the Dual-Luciferase Reporter Assay kit and 216 measured with the Glomax Explorer (both Promega, Madison, WI, USA). Relative luciferase 217 activity was obtained by dividing the relative light units (RLU) produced by the firefly luciferase 218 pGL3 construct by the RLUs produced by Renilla luciferase control reaction. Light generated by 219 the reaction can be correlated with the amount of luciferase protein produced which in turn is 220 proportional to promoter activity driving the gene's expression. Values from an $n=6$ were 221 averaged and normalized to that of the reference construct, pGL3-1093_-31 to obtain a relative 222 measure of activity.

223 Statistical analysis

224 All data were expressed as mean $\pm \mathrm{SD}$. Data from the luciferase assay represents two 225 independent experiments with triplicate measurements. Differences between groups were 226 examined for statistical significance using Student's $t$-test. A $P$-value $<0.01$ denoted the presence 227 of a statistically significant difference. 


\section{Results}

230

231 Clinical Characterization of CHM

232 Family 1

233 We investigated a progressive retinal degeneration in a Caucasian family of American origin 234 (C127). Remarkably, the proband 5116 was the offspring of a consanguineous union between 235 second cousins, with an affected father and carrier mother. To our knowledge, this has never 236 been reported with choroideremia, and it necessitated a thorough and accurate diagnosis of X237 linked CHM and exclusion of an autosomal recessive, or even an unusual male-male 238 transmission as cause for the disease. An investigation of the extended family for which the 239 pedigree is reported in Figure 2A however, clearly demonstrated the X-linked inheritance of the 240 disease.

241 Patient 5116 was initially seen at age 56 years, when he was referred by a retinal specialist with a 242 diagnosis of choroideremia. At his most recent examination, age 76, best corrected visual acuity 243 (BCVA) was measured as 6/19 OD and 6/15 OS. Visual fields were reduced to less than 5 244 degrees. The full-field ERG showed non-detectable dark adapted rod-driven responses as well as 245 non-detectable cone responses to a $30 \mathrm{~Hz}$ flicker stimulus. OCT imaging indicated loss of the 246 photoreceptor layer across the periphery of the fundus with only a small island of RPE remaining 247 in the macula. Posterior segment examination showed a hypo-pigmented fundus with significant 248 atrophy of the RPE and choroid with areas of bare sclera (Figure 3). These findings are 249 consistent with an advanced state of choroideremia. 
Promoter mutation causing choroideremia

250 The proband's daughter 5113 was examined at 33 years of age and did not report any vision 251 difficulties. BCVA when examined was 6/6, both eyes. Full-field ERG testing of the left eye 252 showed dark adapted rod-driven responses were reduced by $50 \%$ in amplitude $(42.5 \mu \mathrm{V})$ and a 253 normal b-wave implicit time ( $81.6 \mathrm{msec})$. Light-adapted cone-driven responses to a $30 \mathrm{~Hz}$ flicker 254 stimulus were reduced by $50 \%$ in amplitude $(27.6 \mu \mathrm{V})$ and borderline reduced in implicit time 255 (25.6 msec). These findings were consistent with a classic carrier state of choroideremia.

256 The proband's father and one brother 5149 were reported to have been previously diagnosed 257 with choroideremia, while a second brother 5147 was reported to be unaffected. Findings are 258 summarized in Table 1.

259 Family 2:

260 Family 2 (GC406) was a Caucasian family of British origin with a history of choroideremia. 261 Proband 111 was first examined at age 13, displaying a reduced but not delayed full field ERG 262 with flicker and bright flash responses both within normal limits. He remained asymptomatic 263 until age 32. Confrontational visual field testing at age 35 showed bilateral infratemporal 264 scotoma, and BCVA was $6 / 5$ in both eyes. He is considered symptomatically mild with retained 265 central macular structure.

266 Of his two maternal uncles, 151 was diagnosed at age 12. Fundus abnormalities typical of 267 choroideremia were noted. 161 was diagnosed at age 8, and upon examination at age 37 268 exhibited moderately constricted visual fields particularly in the superior field bilaterally. BCVA 269 was reduced to count fingers vision OD and 6/36 OS. Fundus examination was consistent with a 270 clinical diagnosis of choroideremia. The pedigree is reported in Figure 2B, and clinical findings 271 summarized in Table 1. 
Promoter mutation causing choroideremia

\section{Genetic Analysis}

$274 \quad$ Family 1 (C127)

275 Genetic analysis of proband 5116 did not reveal any pathogenic mutation in the coding sequence 276 of the $C H M$ gene or splice site boundaries. Yet at position c.-98 relative to the translation start 277 site, a hemizygous C>A transversion was detected (hg38, chrX:g.86047629G>T NM_000390.3, 278 c. $-98 \mathrm{C}>\mathrm{A})$. The variant was not listed in the latest release of dbSNP 279 [http://www.ncbi.nlm.nih.gov/SNP/; accessed Feb 2017] (Sherry et al., 2001). The proband's 280 affected brother (5149) was also found to harbour the variant while it was absent in the 281 unaffected brother (5147) available for testing. The obligate carrier status was confirmed in the 282 proband's daughter (5113). The location of the variant strongly suggested a regulatory mutation, 283 as evaluation of entries in the HGMD reveals that most promoter mutations are located between $284+50$ and -500 from the TSS of a gene (Stenson et al., 2014).

285 Family 2 (GC406):

286 Prior genetic analysis of the coding exons of the CHM gene did not reveal a pathogenic 287 mutation. Whole genome sequencing was performed on proband 111 and his parents 190 and 288 192, as part of the 100,000 Genomes Project. After variant filtering, no causative rare coding 289 variants were identified in any retinal disease gene.

290 In light of the clinical diagnosis of choroideremia in the family, the complete CHM gene was 291 interrogated for rare variants $(\leq 0.001 \mathrm{MAF}$ in 1 Kgenome project and internal cohort of over 2922000 whole genome sequencing samples) hemizygous in the proband and carried by his mother. 293 One such variant was identified, the transition, c.-98C $>$ T (hg38, chrX:g.86047629G $>$ A 294 NM_000390.3, c.-98C>T). The variant was confirmed in the two affected maternal uncles 151 295 and 161 by direct Sanger sequencing. 
Promoter mutation causing choroideremia

296

297 Molecular diagnosis of choroideremia in 5116

298 Immunoblot analysis of protein harvested from a cell line derived from patient 5116 failed to 299 detect the $C H M$ gene product REP-1, providing conclusive confirmation of the clinical diagnosis 300 of choroideremia (Figure 4A). To provide evidence for a regulatory mutation and subsequently 301 reduced transcription, we intended to compare the level of expression between normal and 302 patient samples through qPCR. Endpoint PCR from a cDNA template; however, failed to 303 amplify the 15 exon, 2200 base pair transcript, indicating it was absent or present at a level 304 below the detection threshold, and thus the quantitative assay was not performed. To largely rule 305 out the possibility of a splice defect, we also attempted to amplify a minimal portion of the 306 transcript, a 93 base pair fragment from the 5'-UTR to a region spanning the boundary of exon 1 307 and 2, and found the patient's cells lacked even this short fragment (Figure 4B). A control 308 housekeeping gene was nevertheless readily detected and both partial and full length transcripts 309 were amplified from normal cDNA.

310

311 Effect of the c. $-98 C>A$ and $\underline{c .-98 C}>T$ mutations on transcription of CHM

312 As a starting point, an approximately $1 \mathrm{~kb}$ fragment upstream of the TSS was assayed for ability 313 to drive gene expression, since the majority of elements necessary for transcription are expected 314 to be found within this region (Rockman and Wray, 2002). Comparing the robust luciferase 315 activity produced by cells transfected with this wild type construct pGL3-1093_-31, to that of 316 pGL3-1093_-31 c.-98C $>$ A and c. $-98 \mathrm{C}>\mathrm{T}$, we observed complete abrogation of promoter activity 317 (Figure 1B) in the mutants. The drop from $100 \pm 9.5$ to $2.0 \pm 0.3$ and $1.3 \pm 0.4$ respectively, as 318 measured in normalized relative light units $(\mathrm{RLU}) \pm 1$ standard deviation is even significantly 
319 lower than that of the negative control pGL3-basic which does not contain a promoter sequence, 320 reading at $5.8 \pm 0.9$. This startling observation strongly suggested that the mutation spans an 321 element essential to promoter activity.

322 Based on unpublished reports suggesting other activating elements may exist up to $2.8 \mathrm{~kb}$ 323 upstream of the TSS (Kaiser NW, et al. IOVS 2004;45:ARVO E-Abstract 2451), we additionally 324 compared the activity of two larger constructs approximately $4 \mathrm{~kb}$ and $2 \mathrm{~kb}$ long. Typically, 325 regulatory regions upstream of core promoter sequences contain multiple TF specific binding 326 motifs, where several copies of the same factor or cooperation between different factors serve to 327 synergistically stimulate transcriptional activity of a given gene (Maston et al., 2006). Yet in the 328 case of $C H M$, the longer sequences did not stimulate additional luciferase expression. pGL3329 3983_-31 and pGL3-2027_-31 did not significantly differ from pGL3-1093_-31, producing 83.7 $330 \pm 4.9$ and $96.2 \pm 7.6$ RLUs respectively. The somewhat diminished expression as compared to 331 the reference $1 \mathrm{~kb}$ construct may be attributed to a lower molar amount of the larger plasmids 332 being delivered during transfection.

334 Characterization of CHM promoter

335 To further characterize the promoter, we proceeded to assay progressively shorter constructs, 336 deleting sequences from the $-5^{\prime}$ and $-3^{\prime}$ of the $1 \mathrm{~kb}$ construct to define its boundaries (Figure 1A). 337 Working from the 5' end, the approximately 400, 300, and 100 base pair constructs pGL3-437_338 31, pGL3-346_-31, pGL3-119_-31 did not significantly differ from the $1 \mathrm{~kb}$ containing reference 339 plasmid pGL3-1093_-31, reading at 100.2 $\pm 11.4,96.1 \pm 10.6$, and 97.7 \pm 8.1 RLU respectively. 340 Yet upon deleting a further 11 base pairs, we observed a significant drop to $39.8 \pm 7.6$ RLU with 341 pGL3-108_-31. Having delineated the -5 ' boundary of the promoter as extending to no further 
342 than nucleotide c.-119, we subsequently tested pGL3-119_-76 bearing a deletion from the -3 ' 343 end, and found no significant difference at $93.4 \pm 14$ RLU. It was the final construct, pGL3344 119_-82, which yielded significantly lower activity producing only $27.4 \pm 4$ RLU. Though 345 further characterization of the promoter region down to the single nucleotide level could be 346 performed, we have defined the borders of the promoter to within the region of c.-119 to c.-76.

347 This short 44 base pair DNA region upstream of the $C H M$ coding sequence is able to stimulate 348 transcription in an in-vitro assay at a level that is not significantly different from that of a $1 \mathrm{~kb}$, or 349 even a $4 \mathrm{~kb}$ fragment and can be understood to contain essential cis-acting elements that 350 positively regulate $C H M$ expression.

352 Discussion

353 To date, all genetic defects causing choroideremia, which include all major mutation classes, 354 have been observed in exons, introns or intron/exon boundaries of the CHM gene (Fokkema et 355 al., 2011). No mutations have been reported in the promoter region of $C H M$, and the regulation 356 of the gene has remained essentially unexplored. In this study, we present the first report of 357 promoter mutations, c. $-98 \mathrm{C}>\mathrm{A}$ and c. $-98 \mathrm{C}>\mathrm{T}$, causing choroideremia. The critical role of this 358 residue for gene expression is highlighted by the complete abrogation of reporter activity in a 359 promoter assay when the nucleotide is mutated. Though we demonstrate the first examples in a 360 novel mutation class, it is worthwhile noting that the phenotypes observed in affected individuals 361 were typical of choroideremia. In family 1, the genotype c.-98C $>\mathrm{A}$ in proband 5116 resulted in 362 severe retinopathy, while characteristic milder signs were found in the carrier female 5113.

363 Males from family 2 bearing the c. $-98 \mathrm{C}>\mathrm{T}$ mutation manifested with chorioretinal disease, but 364 with varying degrees of severity; proband 111 remained symptomatically mild while in his 
365 maternal uncles 151 and 161, disease progression was more typical. CHM is known to exhibit a 366 wide interfamilial, and also intrafamilial variability (Moosajee et al., 2014). As the mutations c.$36798 \mathrm{C}>\mathrm{A}$ and c. $-98 \mathrm{C}>\mathrm{T}$ appear to completely abolish transcription, producing no detectable levels 368 of $C H M$ mRNA and REP-1 protein in patient cells, it is not surprising for the disease to present 369 in a classical manner consistent with phenotypes observed for the loss of function mutations. 370 Whole genome sequencing in the parent-offspring trio of family 2 allowed the exclusion of a 371 deep intronic CHM mutation, as well as mono or biallelic mutations in any other retinal 372 dystrophy gene as a cause of the observed symptoms. We therefore conclude that the two 373 mutations at residue c.-98 impair $C H M$ transcription enough to result in choroideremia. For the 374 several gene therapy trials currently underway employing a "gene replacement" strategy 375 (Dimopoulos et al., 2015), individuals bearing these genotypes would be suitable candidates. The 376 two variants have been submitted and published in the LOVD Retinal and Hearing Impairment Genetic Mutation Database (Fokkema et al., 2011).

379 Having identified mutations that evidently abolished transcription, we interpreted their location 380 to span a region crucial for gene expression, and set out to define the boundaries of the gene's 381 promoter. Surprisingly, after analyzing fragments as long as $4 \mathrm{~kb}$, we found a short 44 base pair 382 DNA fragment to be wholly responsible for driving expression. The region c.-119 to c.-76 383 comprises the entirety of the $C H M$ proximal promoter, and is able to drive robust transcription in 384 an in-vitro luciferase assay. The region implicated amounts to less than $3 \%$ of the length of the $385 C H M$ coding sequence where the large majority of reported causative mutations have been found 386 (Fokkema et al., 2011). A recent investigation of a large disease cohort (74), found causative 387 mutations in the gene in $94 \%$ of cases previously diagnosed with CHM (Simunovic et al., 2016). 
Promoter mutation causing choroideremia

388 The remaining $6 \%$ can be understood to be comprised of regulatory mutations, incorrect 389 diagnoses or deep intronic variant causing cryptic splicing (Carss et al., 2017). Promoter defects 390 are therefore expected to be responsible for a small minority of choroideremia cases. The 391 identified region; however, becomes an obvious area for examination in patients in whom no 392 coding sequence mutation is found.

393

394 A promoter or regulatory mutation can be expected to either increase or decrease transcriptional 395 activity mediated by the altered binding capacity of trans-acting protein factors specific to a 396 DNA sequence in the promoter region. In this case, the interaction appears to be entirely 397 disrupted based on the null expression of a reporter driven by mutated sequence in our luciferase 398 assay. Impaired transcription due to a 1-bp mutation in a promoter region is unusual, but has 399 been reported previously as in the case of single base mutations stimulating additional 400 transcriptional activity at the OVOL2 promoter causing autosomal-dominant corneal endothelial 401 dystrophies (Davidson et al., 2016). On the other hand, decreased transactivation of NMNAT1 402 due to a single nucleotide change in the promoter was found to cause Leber congenital amaurosis 403 (Coppieters et al., 2015).

404

405 While we delineate the CHM promoter boundary to this small area upstream of the gene, we 406 cannot exclude the possibility of distant enhancer, repressor, or intronic elements also 407 contributing to regulation; our findings suggest the sequence between c.-119 and c.-76 is 408 essential, but not necessarily sufficient for transcription. The wider upstream sequence of $C H M$ 409 lacks the consensus sequences often found in RNA polymerase II promoters, such as CAAT and 410 TATA boxes; as well it is neither $\mathrm{GC}$ rich nor associated with any $\mathrm{CpG}$ islands. Bioinformatic 
411 analysis with MotifMap, a dataset of computationally predicted transcription factor binding sites

412 based on binding motifs [http://motifmap.ics.uci.edu/] (Daily et al., 2011) identifies a putative

413 binding motif for the transcription factor zinc finger protein 143 (ZNF143) contained within the

414 region of the now revealed $C H M$ promoter. ZNF143 participates in the regulation of RNA pol II

415 and III mediated transcription of protein coding, non-coding, and small nuclear RNA (Schaub et

416 al., 1997; Myslinski et al., 1998) and was initially connected with the binding motif

417 TTCCCATTATGCACCGCG (SBS1) (Myslinski et al., 2006). Genome-wide studies revealed

418 the binding site for the factor to be frequently found with an adjacent 5' accessory sequence,

419 forming the ACTACAATTCCCATTATGCACCGCG (SBS2) motif. SBS2 is comprised of both

420 a THAP domain-containing protein 11 (THAP11) and ZNF143 binding site, with the factors

421 believed to act in a competitive manner (Ngondo-Mbongo et al., 2013). The recruitment of

422 THAP11 to its canonical binding site ACTAYRNNNCCCR is most frequently associated with

423 up-regulation genes essential to protein biosynthesis and energy production (Dejosez et al.,

424 2010). More recently, ZNF143 was suggested to cooperatively occupy SBS2 sites with THAP11

425 and a third factor, the scaffold protein host cell factor 1 (HCFC1) in-vivo (Vinckevicius et al.,

426 2015). SBS2 is closely matched by the sequence found at position c.-108 to c.-84 upstream of

427 CHM, ACTACAACACCCAGAATGCACTGTT. Notably, ZNF143's binding at promoters was

428 recently implicated in chromatin looping with distal regulatory elements (Bailey et al., 2015),

429 suggesting the involvement of yet other factors in the total regulation of expression of CHM.

430

431 Of the three transcription factors implicated above, the binding of at least ZNF143 is supported

432 by publicly available chromatin immunoprecipitation sequencing data released as part of the

433 ENCODE project [https://genome.ucsc.edu/ENCODE/] (ENCODE Project Consortium, 2012). 
434 In all cell types assayed: lymphoblastoid, HeLa and K562 cell lines, as well as embryonic stem 435 cells, the promoter region of $C H M$ interacted with ZNF143, for which a consensus binding 436 sequence GAACTACAATTCCCAGAAGGC, again is closely matched by 437 GAACTACAACACCCAGAATGC found between position c.-110 toc.-91 relative to the A of 438 the CHM start codon. The position-weight matrix for ZNF143 (Figure 5) establishes the relative 439 frequency of the base $\mathrm{C}$ at position c. -98 to be $100 \%$, supporting the importance of the residue 440 and the resulting pathogenesis when mutated. Furthermore, surveying the UCSC genome 441 browser (http://genome.ucsc.edu/) multiz alignment of 100 vertabrate genomes tract, the region c.-119 to c. -76 shows a high degree of conservation among mammals, and absolute conservation of residue $\mathrm{C}$ at positions corresponding to c. -98 pointing to an important biological role for the sequence (Kuhn et al., 2007; Blanchette et al., 2004). Representatives of birds and amphibian classes, however, lack homology in the region, in while in fish a corresponding region is absent 446 altogether. The promoter, therefore, cannot be considered an ultra-conserved non-coding element (Dimitrieva and Bucher, 2013). Several alignments are listed in Table 2.

449 Studies of $C H M$ mRNA and protein localization have found a broad expression profile for both.

450 In mice, evidence of transcription was found in multiple cell types and in every major layer of 451 retina (Keiser et al., 2005), while immunolabeling of primate retina showed REP-1 localized to 452 both rod and cones (Dimopoulos et al., 2015). Studies in human and primate retina found that 453 mRNA levels did not correspond to the pattern of disease expression; little $C H M$ was detected in 454 the RPE and choroid, and there were no marked regional differences in the concentration of 455 CHM mRNA apparent with foveal versus mid-peripheral total RNA despite affected males 456 typically exhibiting a preservation of central vision until late in the disease (Bernstein and Wong, 
457 1998). Additionally, REP-1 can be readily detected in human fibroblasts or peripheral blood 458 mononuclear cells (Furgoch et al., 2014; MacDonald et al., 1998). Taken together with 459 ZNF143's characterization as one of the most common and ubiquitously expressed TFs 460 (Myslinski et al., 1998) a picture emerges of widespread and non-specific transcription of CHM, 461 despite choroideremia's manifestation as an ocular disease. Indeed, patients' apparent lack of 462 systemic symptoms can be understood to result not from tissue specific expression of REP-1, but 463 from the differing affinities of REP-1 and REP-2 for target Rabs, which may themselves be 464 differentially expressed or possess tissue or cell specific activity. Investigators have implicated 465 Rab27 (Seabra et al., 1995) and Rab38 (Kohnke et al., 2013) as possible contributors.

467 The study presented here also poses interesting questions, such as whether mutations of other 468 residues less critical to transactivator binding in the $C H M$ promoter, that diminish, but not 469 completely abolish mRNA expression, can result in a milder phenotype, or a different rate of 470 progression of choroideremia. Currently, the dbSNP database lists no known SNPs between c.471119 to c.-76 (Sherry et al., 2001). Having shown the $C H M$ region responsible for regulation of its 472 expression, described for the first time the features of its promoter, and extended the inventory of 473 molecular changes causing choroideremia, the findings are of clinical and diagnostic interest and 474 present an obvious area of examination for patients with CHM in whom no coding sequence 475 mutation has been found. Further elucidating the roles of ZNF143, THAP11, HCFC1 or other 476 distal factors will prove an important step toward understanding the complete picture of $C H M$ 's 477 regulation. 
Promoter mutation causing choroideremia

480 The authors have no proprietary or commercial interest in any materials discussed in this article.

481 Support by Canadian Institutes of Health Research, Emerging Team Grant: 119190, Foundation

482 Fighting Blindness, Canada, Choroideremia Research Foundation Canada, Inc., and Alberta

483 Innovates-Health Solutions 201201139 is acknowledged (IM).

484 This project was also supported by The National Institute for Health Research (NIHR) and 485 Biomedical Research Centre (BRC) at Moorfields Eye Hospital and the UCL Institute of 486 Ophthalmology (GA, AW).

487 This research was made possible through access to the data and findings generated by the 488 100,000 Genomes Project. The 100,000 Genomes Project is managed by Genomics England 489 Limited (a wholly owned company of the Department of Health). The 100,000 Genomes Project 490 is funded by the NIHR and NHS England. The Wellcome Trust, Cancer Research UK and the 491 Medical Research Council have also funded research infrastructure.

492

493 Support by the National Eye Institute grant EY-09076, and Foundation Fighting Blindness is also 494 acknowledged (DB)

495

496

497

498

$499 \quad$ Figure Legends

500 Figure 1. Functional Analysis of the $C H M$ Promoter. 
501 (A) The constructs portrayed on the left were inserted upstream of the luciferase gene in pGL3502 basic. Nucleotide +1 represents the translation start site. The promoterless pGL3-basic served as 503 a negative control. (B) The mutations c. $-98 \mathrm{C}>\mathrm{A}$, and $\underline{\mathrm{c} .-98 \mathrm{C}}>\mathrm{T}$ abolish transcription, while the 504 minimal construct c.-119_-76 is sufficient for robust expression of the reporter gene not 505 significantly different from even that of the nearly $4 \mathrm{~kb}$ construct. All constructs were transiently 506 transfected into HEK293T cells. A dual-luciferase reporter assay was used to assess the potential 507 promoter activity of various sized inserts and the c.-98 mutants. Promoter activity is shown as a 508 ratio of firefly luciferase over Renilla luciferase present on the transfection control plasmid pRL509 CMV to account for inter-well variation. Activity is normalized to that of the reference construct 510 pGL3-1093_-31 which is artificially set to equal 100. Activity significantly different $(\mathrm{P}<0.01)$

511 from the reference construct is denoted by an asterisk. Error bars represent $\pm 1 \mathrm{SD}$.

512 Figure 2. Pedigree Structure of Affected Families with CHM Promoter Mutations

513 (A) Family1. The parents of proband 5116 were second cousins, sharing a set of great grand514 parents. The inheritance pattern mimics male-male transmission, but is nevertheless consistent 515 with X-linked inheritance upon examination of the wider family pedigree. (B) Family 2. The 516 pedigree showing three generations affected by choroideremia examined in this study.

517 Inheritance follows an X-linked pattern.

$518 \quad$ Figure 3. Retinal features in patient 5116.

519 Legend: Left column (OD), right column (OS). (A) Fundus photographs of the proband taken at 520 age 76 showing typical choroideremia changes, with atrophy of the choroid and RPE. A small 521 island of preserved RPE remains in the central macula, surrounded by atrophic peripheral areas 522 of apparent bare sclera. (B)(C) Fundus autofluorescence image demonstrating areas of residual 523 RPE (L) and the corresponding OCT image (R). Preserved retinal areas with normal 
524 autofluorescence exhibit thicker choroid and preserved retinal lamination. An outer retinal

525 tubulation is seen in the right fundus.

526 Figure 4. Molecular confirmation of choroideremia in patient 5116.

527 (A) Patient 5116 lacks REP-1. Western blot results show the absence of a $\sim 100 \mathrm{kDa}$ band 528 corresponding to REP-1 in a lymphoblastoid cell line derived from the patient (lane 2), which is 529 present in a normal control (lane 1). A $\beta$-actin antibody was used as a loading control to ensure 530 an adequate protein sample in each lane, with the $42 \mathrm{kDa}$ band present in both samples.

531 (B) Patient 5116 lacks CHM mRNA. cDNA synthesized from the mRNA harvested from a 532 patient generated lymphoblastoid cell line was used as template for PCR. Lanes 2 and 3 show a $533475 \mathrm{bp}$ band resulting from the amplification of the GAPDH control housekeeping gene from 5345116 and a normal control, indicating cDNA of adequate quality. Lanes 6 and 9 demonstrate an 535 absence of amplification from the patient's cDNA of both partial and full length coding 536 sequence, respectively, as compared to PCR products sized 93 and 2200 base pairs amplified 537 from normal cDNA observed in lanes 5 and 10.

538 Figure 5. Consensus binding sequences for transcription factor ZNF143.

539 (A) Partial map of the human $C H M$ gene; arrow indicates transcription start site. (B) Expanded 540 sequence of the minimal $C H M$ promoter from c.-119 to c.-76, as identified through the analysis 541 of progressive deletion constructs. Position c.-98 is marked with an asterisk. (C) Sequence logo 542 derived from publically available ChIP-seq data released as part of the ENCODE project, with 543 the position weighted matrix below (ENCODE Project Consortium, 2012). An invariant $\mathrm{C}$ is 544 found at position corresponding to c.-98 of $\mathrm{CHM}$. 545

546 Table 1. Clinical data of genotyped individuals 
547

$\underline{\text { Table 2. Multiz alignment of the promoter region of } 15 \text { CHM orthologs }}$

548

549 References

550 Anderson MA, Gusella JF. 1984. Use of cyclosporin A in establishing epstein-barr virus-

551 transformed human lymphoblastoid cell lines. In Vitro 20:856-858.

552 Bailey SD, Zhang X, Desai K, Aid M, Corradin O, Cowper-Sal Lari R, Akhtar-Zaidi B, Scacheri

553 PC, Haibe-Kains B, Lupien M. 2015. ZNF143 provides sequence specificity to secure chromatin 554 interactions at gene promoters. Nat Commun 2:6186.

555 Bernstein SL, Wong P. 1998. Regional expression of disease-related genes in human and 556 monkey retina. Mol Vis 4:24.

557 Blanchette M, Kent WJ, Riemer C, Elnitski L, Smit AF, Roskin KM, Baertsch R, Rosenbloom

558 K, Clawson H, Green ED, Haussler D, Miller W. 2004. Aligning multiple genomic sequences 559 with the threaded blockset aligner. Genome Res 14:708-715.

560 Carss KJ, Arno G, Erwood M, Stephens J, Sanchis-Juan A, Hull S, Megy K, Grozeva D,

561 Dewhurst E, Malka S, Plagnol V, Penkett C, Stirrups K, Rizzo R, Wright G, Josifova D, Bitner-

562 Glindzicz M, Scott RH, Clement E, Allen L, Armstrong R, Brady AF, Carmichael J, Chitre M,

563 Henderson RH, Hurst J, MacLaren RE, Murphy E, Paterson J, Rosser E, Thompson DA,

564 Wakeling E, Ouwehand WH, Michaelides M, Moore AT, NIHR-BioResource Rare Diseases

565 Consortium, Webster AR, Raymond FL. 2017. Comprehensive rare variant analysis via whole-

566 genome sequencing to determine the molecular pathology of inherited retinal disease. Am J Hum

567 Genet 100:75-90. 
Promoter mutation causing choroideremia

568 Chi JY, MacDonald IM, Hume S. 2013. Copy number variant analysis in CHM to detect

569 duplications underlying choroideremia. Ophthalmic Genet 34:229-233.

570 Coppieters F, Todeschini AL, Fujimaki T, Baert A, De Bruyne M, Van Cauwenbergh C, Verdin

571 H, Bauwens M, Ongenaert M, Kondo M, Meire F, Murakami A, Veitia RA, Leroy BP, De Baere

572 E. 2015. Hidden genetic variation in LCA9-associated congenital blindness explained by 5'UTR

573 mutations and copy-number variations of NMNAT1. Hum Mutat 36:1188-1196.

574 Coussa RG, Traboulsi EI. 2012. Choroideremia: A review of general findings and pathogenesis.

575 Ophthalmic Genet 33:57-65.

576 Cremers FP, Armstrong SA, Seabra MC, Brown MS, Goldstein JL. 1994. REP-2, a rab escort

577 protein encoded by the choroideremia-like gene. J Biol Chem 269:2111-2117.

578 Cremers FP, van de Pol DJ, van Kerkhoff LP, Wieringa B, Ropers HH. 1990. Cloning of a gene

579 that is rearranged in patients with choroideraemia. Nature 347:674-677.

580 Daily K, Patel VR, Rigor P, Xie X, Baldi P. 2011. MotifMap: Integrative genome-wide maps of 581 regulatory motif sites for model species. BMC Bioinformatics 12:495-2105-12-495.

582 Davidson AE, Liskova P, Evans CJ, Dudakova L, Noskova L, Pontikos N, Hartmannova H, 583 Hodanova K, Stranecky V, Kozmik Z, Levis HJ, Idigo N, Sasai N, Maher GJ, Bellingham J, Veli 584 N, Ebenezer ND, Cheetham ME, Daniels JT, Thaung CM, Jirsova K, Plagnol V, Filipec M, 585 Kmoch S, Tuft SJ, Hardcastle AJ. 2016. Autosomal-dominant corneal endothelial dystrophies 586 CHED1 and PPCD1 are allelic disorders caused by non-coding mutations in the promoter of 587 OVOL2. Am J Hum Genet 98:75-89. 
588 de Vooght KM, van Wijk R, van Solinge WW. 2009. Management of gene promoter mutations

589 in molecular diagnostics. Clin Chem 55:698-708.

590 Dejosez M, Levine SS, Frampton GM, Whyte WA, Stratton SA, Barton MC, Gunaratne PH, 591 Young RA, Zwaka TP. 2010. Ronin/hcf-1 binds to a hyperconserved enhancer element and 592 regulates genes involved in the growth of embryonic stem cells. Genes Dev 24:1479-1484.

593 Dimitrieva S, Bucher P. 2013. UCNEbase--a database of ultraconserved non-coding elements 594 and genomic regulatory blocks. Nucleic Acids Res 41:D101-9.

595 Dimopoulos IS, Chan S, MacLaren RE, MacDonald IM. 2015. Pathogenic mechanisms and the 596 prospect of gene therapy for choroideremia. Expert Opin Orphan Drugs 3:787-798.

597 ENCODE Project Consortium. 2012. An integrated encyclopedia of DNA elements in the human 598 genome. Nature 489:57-74.

599 Esposito G, De Falco F, Tinto N, Testa F, Vitagliano L, Tandurella IC, Iannone L, Rossi S, 600 Rinaldi E, Simonelli F, Zagari A, Salvatore F. 2011. Comprehensive mutation analysis (20 601 families) of the choroideremia gene reveals a missense variant that prevents the binding of REP1 602 with rab geranylgeranyl transferase. Hum Mutat 32:1460-1469.

603 Fokkema IF, Taschner PE, Schaafsma GC, Celli J, Laros JF, den Dunnen JT. 2011. LOVD v.2.0:

604 The next generation in gene variant databases. Hum Mutat 32:557-563.

605 Freund PR, Sergeev YV, MacDonald IM. 2016. Analysis of a large choroideremia dataset does 606 not suggest a preference for inclusion of certain genotypes in future trials of gene therapy. Mol 607 Genet Genomic Med 4:344-358. 
608 Furgoch MJB, Mewes-Arès J, Radziwon A, MacDonald IM. 2014. Molecular genetic diagnostic 609 techniques in choroideremia. Mol Vision 20:535-544.

610 Karna J. 1986. Choroideremia. A clinical and genetic study of 84 finnish patients and 126 female 611 carriers. Acta Ophthalmol Suppl 176:1-68.

612 Keiser NW, Tang W, Wei Z, Bennett J. 2005. Spatial and temporal expression patterns of the 613 choroideremia gene in the mouse retina. Mol Vision 11:1052-1060.

614 Kohnke M, Delon C, Hastie ML, Nguyen UT, Wu YW, Waldmann H, Goody RS, Gorman JJ, 615 Alexandrov K. 2013. Rab GTPase prenylation hierarchy and its potential role in choroideremia 616 disease. PLoS One 8:e81758.

617 Kuhn RM, Karolchik D, Zweig AS, Trumbower H, Thomas DJ, Thakkapallayil A, Sugnet CW, 618 Stanke M, Smith KE, Siepel A, Rosenbloom KR, Rhead B, Raney BJ, Pohl A, Pedersen JS, Hsu 619 F, Hinrichs AS, Harte RA, Diekhans M, Clawson H, Bejerano G, Barber GP, Baertsch R, 620 Haussler D, Kent WJ. 2007. The UCSC genome browser database: Update 2007. Nucleic Acids 621 Res 35:D668-73.

622 MacDonald IM, Mah DY, Ho YK, Lewis RA, Seabra MC. 1998. A practical diagnostic test for 623 choroideremia. Ophthalmology 105:1637-1640.

624 Maston GA, Evans SK, Green MR. 2006. Transcriptional regulatory elements in the human 625 genome. Annu Rev Genomics Hum Genet 7:29-59.

626 McTaggart KE, Tran M, Mah DY, Lai SW, Nesslinger NJ, MacDonald IM. 2002. Mutational 627 analysis of patients with the diagnosis of choroideremia. Hum Mutat 20:189-196. 
628 Moosajee M, Ramsden SC, Black GC, Seabra MC, Webster AR. 2014. Clinical utility gene card 629 for: Choroideremia. Eur J Hum Genet 22:10.1038/ejhg.2013.183. Epub 2013 Aug 21.

630 Myslinski E, Gerard MA, Krol A, Carbon P. 2006. A genome scale location analysis of human 631 staf/ZNF143-binding sites suggests a widespread role for human staf/ZNF143 in mammalian 632 promoters. J Biol Chem 281:39953-39962.

633 Myslinski E, Krol A, Carbon P. 1998. ZNF76 and ZNF143 are two human homologs of the 634 transcriptional activator staf. J Biol Chem 273:21998-22006.

635 Ngondo-Mbongo RP, Myslinski E, Aster JC, Carbon P. 2013. Modulation of gene expression via 636 overlapping binding sites exerted by ZNF143, Notch1 and THAP11. Nucleic Acids Res 41:40006374014.

638 Roberts MF, Fishman GA, Roberts DK, Heckenlively JR, Weleber RG, Anderson RJ, Grover S. 639 2002. Retrospective, longitudinal, and cross sectional study of visual acuity impairment in 640 choroideraemia. Br J Ophthalmol 86:658-662.

641 Rockman MV, Wray GA. 2002. Abundant raw material for cis-regulatory evolution in humans. 642 Mol Biol Evol 19:1991-2004.

643 Schaub M, Myslinski E, Schuster C, Krol A, Carbon P. 1997. Staf, a promiscuous activator for 644 enhanced transcription by RNA polymerases II and III. Embo J 16:173-181.

645 Seabra MC, Brown MS, Goldstein JL. 1993. Retinal degeneration in choroideremia: Deficiency 646 of rab geranylgeranyl transferase. Science 259:377-381. 
Promoter mutation causing choroideremia

647 Seabra MC, Brown MS, Slaughter CA, Sudhof TC, Goldstein JL. 1992. Purification of

648 component A of rab geranylgeranyl transferase: Possible identity with the choroideremia gene

649 product. Cell 70:1049-1057.

650 Seabra MC, Ho YK, Anant JS. 1995. Deficient geranylgeranylation of ram/Rab27 in

651 choroideremia. J Biol Chem 270:24420-24427.

652 Sergeev YV, Smaoui N, Sui R, Stiles D, Gordiyenko N, Strunnikova N, MacDonald IM. 2009.

653 The functional effect of pathogenic mutations in rab escort protein 1. Mutat Res 665:44-50.

654 Sherry ST, Ward MH, Kholodov M, Baker J, Phan L, Smigielski EM, Sirotkin K. 2001. dbSNP:

655 The NCBI database of genetic variation. Nucleic Acids Res 29:308-311.

656 Simunovic MP, Jolly JK, Xue K, Edwards TL, Groppe M, Downes SM, MacLaren RE. 2016.

657 The spectrum of CHM gene mutations in choroideremia and their relationship to clinical

658 phenotype. Invest Ophthalmol Vis Sci 57:6033-6039.

659 Stenson PD, Mort M, Ball EV, Shaw K, Phillips A, Cooper DN. 2014. The human gene mutation

660 database: Building a comprehensive mutation repository for clinical and molecular genetics,

661 diagnostic testing and personalized genomic medicine. Hum Genet 133:1-9.

662 van Bokhoven H, van den Hurk JA, Bogerd L, Philippe C, Gilgenkrantz S, de Jong P, Ropers

663 HH, Cremers FP. 1994. Cloning and characterization of the human choroideremia gene. Hum

664 Mol Genet 3:1041-1046.

665 Van Den Hurk JAJM, Van De Pol DJR, Wissinger B, Van Driel MA, Hoefsloot LH, De Wijs IJ,

666 Van Den Born I, Heckenlively JR, Brunner HG, Zrenner E, Ropers H-, Cremers FPM. 2003. 
667 Novel types of mutation in the choroideremia (CHM) gene: A full-length L1 insertion and an 668 intronic mutation activating a cryptic exon. Hum Genet 113:268-275.

669 Vinckevicius A, Parker JB, Chakravarti D. 2015. Genomic determinants of

670 THAP11/ZNF143/HCFC1 complex recruitment to chromatin. Mol Cell Biol 35:4135-4146.

671 
Promoter mutation causing choroideremia

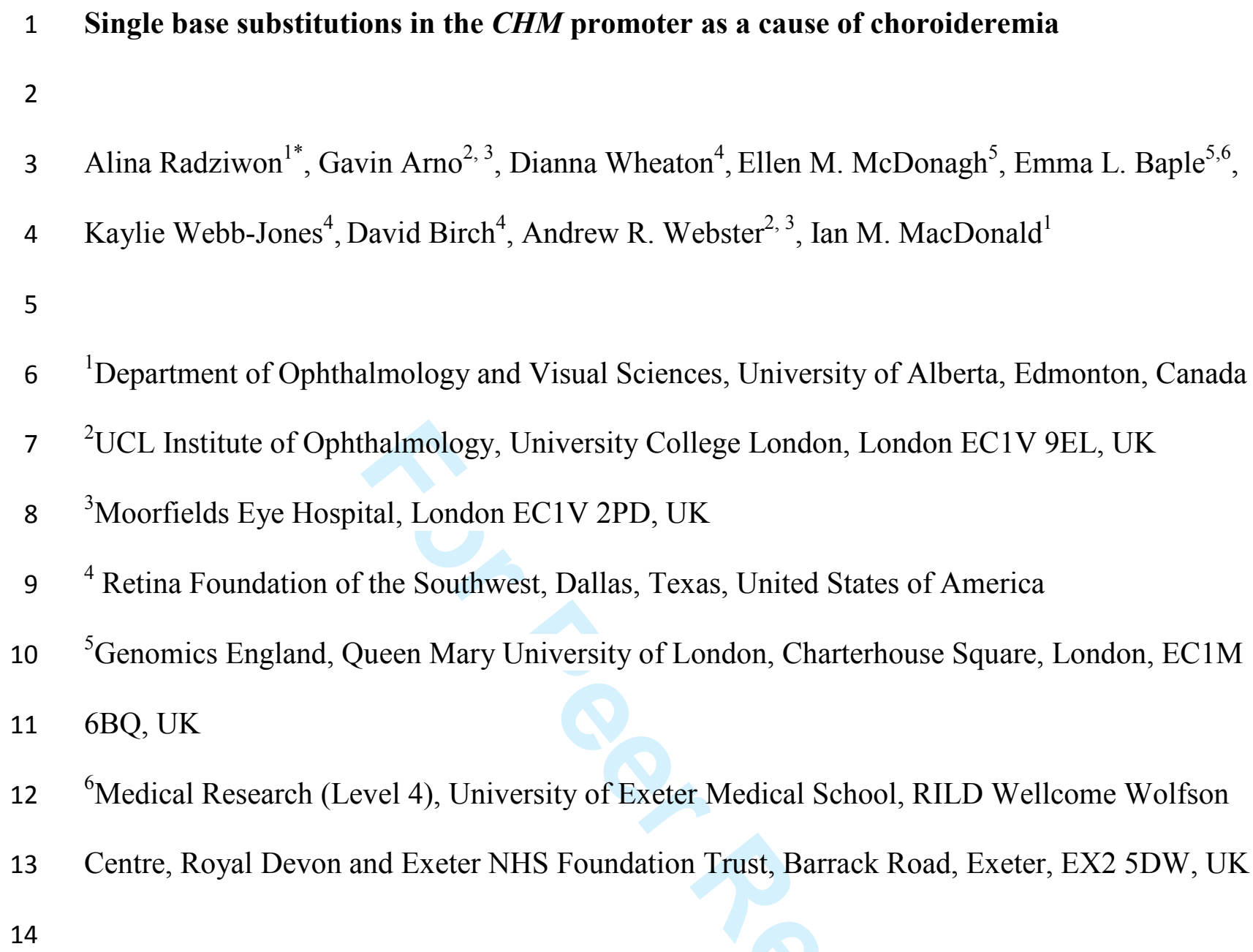


22 Financial Support: IM: Canadian Institutes of Health Research, Emerging Team Grant: 119190,

23 Foundation Fighting Blindness, Canada, Choroideremia Research Foundation Canada, Inc., and

24 Alberta Innovates-Health Solutions 201201139

25 DB: National Eye Institute grant EY-09076, Foundation Fighting Blindness

26 GA, AW: The National Institute for Health Research (NIHR) Biomedical Research Centre

27 (BRC) at Moorfields Eye Hospital, and the UCL Institute of Ophthalmology. This research was

28 made possible through access to the data and findings generated by the 100,000 Genomes

29 Project. The 100,000 Genomes Project is managed by Genomics England Limited (a wholly

30 owned company of the Department of Health). The 100,000 Genomes Project is funded by the

31 NIHR and NHS England. The Wellcome Trust, Cancer Research UK and the Medical Research

32 Council have also funded research infrastructure. 
Promoter mutation causing choroideremia

\begin{abstract}
45 Abstract
46 Although over 150 unique mutations affecting the coding sequence of $C H M$ have been identified

47 in patients with the X-linked chorioretinal disease choroideremia (CHM), no regulatory

48 mutations have been reported, and indeed the promoter has not been defined. Here we describe

49 two independent families affected by CHM bearing a mutation outside the gene's coding region

50 at position c.-98: $\mathrm{C}>\mathrm{A}$ and $\mathrm{C}>\mathrm{T}$, which segregated with the disease. The male proband of family

511 was found to lack $C H M$ mRNA and its gene product Rab escort protein 1 (REP-1), while

52 whole genome sequencing of an affected male in family 2 excluded the involvement of any other

53 known retinal genes. Both mutations abrogated luciferase activity when inserted into a reporter

54 construct, and by further employing the luciferase reporter system to assay sequences $5^{\prime}$ to the

55 gene, we identified the CHM promoter as the region encompassing nucleotides c.-119 to c.-76.

56 These findings suggest that the $C H M$ promoter region should be examined in patients with

57 choroideremia who lack coding-sequence mutations, and reveals, for the first time, features of

58 the gene's regulation.

59

60

61

62

63

64

65

66 Keywords

67 CHM, choroideremia, promoter, REP-1, ZNF143, THAP11
\end{abstract}


Promoter mutation causing choroideremia

68

69

70

71

72

73

74

75

76

77

78

79

80

81

82

83

84

85

86

87

88

89

90

\section{Introduction}

Choroideremia (CHM; MIM\# 303100) is an X-linked, recessively inherited chorioretinal dystrophy with an incidence of 1/50,000. Progressive degeneration of photoreceptors, retinal pigment epithelium (RPE), and the choroid causes affected hemizygous males to develop night blindness in the first or second decade of life, followed by a decrease in peripheral visual fields and an eventual loss of central visual acuity in advanced stages of the disease (Coussa and Traboulsi, 2012). Carrier females, while usually asymptomatic, may exhibit signs of retinal degeneration upon fundoscopic examination, and more rarely have reduced dark adaptation and peripheral vision (Karna, 1986; Roberts et al., 2002).

To date, CHM has only been linked to mutations within the CHM gene, coding for REP-1 (Cremers et al., 1990). The protein serves as a molecular chaperone for small GTPases from the Rab family, presenting them to Rab geranylgeranyl transferase which modifies them by the covalent attachment of a lipid moiety. The lipid modification, known as prenylation, of target Rab proteins is essential for intracellular vesicular transport (Seabra et al., 1992; Seabra et al., 1993). A homologue, REP-2, encoded by $C H M L$ or choroideremia-like, functions similarly and appears to compensate for the absence of REP-1 in all tissues except the eye (Cremers et al., 1994). CHM spans over $150 \mathrm{~kb}$ of Xq21.2 and contains 15 exons (van Bokhoven et al., 1994). A 30 base pair 5'untranslated region, found on exon 1, precedes an open reading frame encoding the 653 amino acid REP-1 protein. At least 163 unique pathogenic mutations have been reported

in the LOVD Retinal and Hearing Impairment Genetic Mutation Database [https://grenada.lumc.nl/LOVD2/Usher_montpellier/home.php ; accessed Feb 2017] (Fokkema et al., 2011). The mutation spectrum includes transitions and transversions leading to protein truncation, splice defects, indels and large deletions ranging from a single exon to the full gene. 
91 Missense mutations predicted to alter protein structure or impair function (Sergeev et al., 2009;

92 Esposito et al., 2011), transposon insertions (Van Den Hurk et al., 2003), partial gene

93 duplications (Chi et al., 2013; Simunovic et al., 2016) and other variations are infrequently

94 found, but taken collectively, almost all known pathogenic variants in the CHM gene have been 95 loss of function mutations that abolish functional REP-1 (McTaggart et al., 2002; Simunovic et 96 al., 2016). Notably there is no apparent correlation between genotype and phenotype, with the 97 age at onset of symptoms, visual acuity and visual fields being unrelated to mutation type 98 (Freund et al., 2016; Simunovic et al., 2016).

99 The promoter driving expression of $C H M$ has been heretofore unidentified. Analyses of 100 promoter mutations causing inherited diseases can be useful in identifying transcription factors 101 involved in the regulation and expression of a gene of interest, as the activity of RNA 102 polymerase II is mediated by the recruitment of general and sequence specific transcription 103 factors to cis-acting regulatory sequences. The core promoter directing basal level transcription 104 is generally found between nucleotides -40 and +50 of the transcription start site (TSS) (de 105 Vooght et al., 2009), while proximal promoter elements typically reside within 1 kilo bases (kb), 106 with additional enhancers, repressors, insulators acting even over distances of mega bases 107 (Maston et al., 2006). The investigation of regulatory elements in the context of human disease is 108 complicated by the fact that in contrast to loss of function mutations, regulatory defects may 109 produce small quantitative changes that are difficult to detect. Mutations in cis-acting regulatory 110 sequences are a significant cause of human disease, and according to statistics compiled by the 111 Human Gene Mutation Database [http://www.hgmd.cf.ac.uk ; accessed May 2016] 112 approximately $2 \%$ of disease causing point mutations are in non-coding regions of the genome 113 (Stenson et al., 2014). Though promoter defects can cause functionally important consequences 
114 for gene expression, their analysis is often not a regular part of DNA diagnostics as the 115 investigation can be complex, laborious and difficult to perform.

116 In this study, we report the first known regulatory mutations causing choroideremia. The absence 117 of a genetic defect in a CHM family prompted us to explore the upstream non-coding region, 118 revealing the novel promoter variant c.-98C $>$ A. Subsequently, whole genome sequencing in an 119 unrelated family with CHM identified a second variant of the same residue, c. $-98 \mathrm{C}>\mathrm{T}$. We 120 demonstrate the mutations' effects on transcription using a luciferase assay, and employ the same 121 system to further characterize the boundary of the $C H M$ promoter that spans the location. The 122 results suggest a disruption of a transcription factor binding site and impaired transactivation of 123 the CHM promoter by the factor(s). Together, these studies further our understanding of 124 regulation and expression of $C H M$ and present a possible explanation for cases of unexplained 125 choroideremia where no causative mutation is found within the gene.

127 Materials and Methods

128

129 Clinical Examination and Study Subjects

130 This study was approved by the institutions' respective ethics review boards. All procedures 131 adhered to the tenets of the Declaration of Helsinki. Before participation, the purpose and risks 132 of the study were explained, and informed consent was obtained. Blood samples were drawn, 133 and a detailed pedigree and history was recorded.

134 We studied 4 males affected by CHM and 1 carrier female from two unrelated families. 135 Individuals were examined by fundoscopy and functional ophthalmologic methods including 136 some or all of: visual acuity testing, central visual fields, full field electroretinography, optical 
137 coherence tomography and fundus autofluorescence imaging. Choroideremia was diagnosed by 138 senior clinicians with expertise in inherited retinal disease based on clinical findings alone. 139 Affected males had a history of night vision loss and fundus appearance of peripheral retinopathy 140 with broad areas of RPE and choroidal atrophy.

\section{CHM Sanger Sequencing}

143 DNA was extracted from lymphocytes using conventional methodologies. Proband 5116 from 144 family 1 (C127) was initially screened for $C H M$ mutations in the coding sequence and splice 145 sites by direct Sanger sequencing from PCR amplicons (Furgoch et al., 2014). Additionally, 1kb 146 of the 5'-flanking sequence was amplified with primers 5'-CAGGGAAGGCCCACTACTGC -3' 147 and 5'-CTTGTGGAAATGAGATCAAGTTAGG-3' and sequenced with the same primers. With 148 the exception of patient 111 and his parents, of family 2 who underwent whole genome 149 sequencing, the remaining study individuals were genotyped only at position c.-98 to confirm the 150 presence or absence of the respective variant. Annotation is in accordance with GenBank: 151 NM_000390.3, where +1 represents the start of translation.

\section{Cell Culture}

154 Leukocytes from patient 5116 were separated with Ficoll-Histopaque (Sigma-Aldrich, St. Louis, 155 MO, USA) from $10 \mathrm{~mL}$ of whole human blood collected in acid citrate dextrose (ACD-A) tubes 156 (BD, Franklin Lakes, NJ, USA). A lymphoblastoid cell line was established by Epstein-Barr 157 virus transformation within 48 hours of collection (Anderson and Gusella, 1984). Cells were 158 maintained in RPMI-1640 supplemented with $15 \%$ fetal calf serum and penicillin-streptomycin. 
160

161 Total RNA was extracted from $5 \times 10^{6}$ lymphoblastoid cells from patient 5116 and a healthy 162 control, with the NucleoSpin RNA isolation kit (Macherey-Nagel, Düren, Germany) according to 163 the manufacturer's protocol. cDNA was transcribed from $5 \mu \mathrm{g}$ of total RNA with the RevertAid 164 First Strand cDNA Synthesis Kit (Thermo Scientific, Rockford, IL, USA) using a random 165 hexamer primer mix. PCR amplification from cDNA with forward primer 5'166 TAATAGTCACATGACACGTTTCCCG-3', paired with 5'-TGGATTCAGGCAAACCCGT-3', 167 or 5'-TTTAAAATGAGCAAGTCAATGTGC-3' as a reverse primer was used to detect partial 168 transcript (spanning the junction of exon 1 and 2), or the entire coding sequence of $C H M$ 169 encompassed by 15 exons, respectively. GAPDH was also amplified as a positive control with 170 intron-spanning primers.

171 Protein was extracted from $2 \times 10^{6}$ lymphoblastoid cells and immunoblot analysis of REP-1 was 172 performed as previously described (Furgoch et al., 2014).
174

175 UK proband 111 (GC406) and his unaffected parents 190 and 192 underwent whole genome 176 sequencing (WGS) as part of the 100,000 Genomes Project. Briefly, genomic DNA was 177 processed using the Illumina TruSeq DNA PCR-Free Sample Preparation kit (Illumina Inc) and 178 sequenced using an Illumina HiSeq X Ten, generating minimum coverage of $15 \mathrm{X}$ for $>97 \%$ of 179 the callable autosomal genome. Reads were aligned to build GRCh37 of the human genome 180 using the Isaac aligner (Illumina Inc). SNVs and indels were identified using Platypus v0.8.1 and 181 annotated using Cellbase (https://github.com/opencb/cellbase). Variant filtering was performed 182 using minor allele frequency (MAF) in publicly available and in-house datasets, predicted 
Promoter mutation causing choroideremia

183 protein impact and familial segregation. Surviving variants were prioritized using a pre-specified 184 virtual gene panel from PanelApp (https://panelapp.extge.co.uk/crowdsourcing/PanelApp/ 185 Posterior segment abnormalities v1.7. Allelic state was required to match the curated mode of 186 inheritance for variants in panel genes.

188 Promoter Analysis

189 Construction of luciferase reporter plasmids

190 Primers were initially designed to amplify a $1063 \mathrm{bp}$ fragment from upstream of the CHM 191 transcription start site (c.-1093_c.-31) from control and patient 5116's DNA. The c.-98C >T 192 mutation present in family 2 was generated by site directed mutagenesis from the control 193 plasmid. All sequences cloned contained only untranscribed DNA and did not include the 30 194 base pair 5'UTR directly upstream of the start codon. The fragment was cloned according to 195 standard protocols, with SacI and HindIII restriction sites added to primers allowing digest by 196 the enzymes (Thermo Scientific, Rockford, IL, USA). Ligation into the promoterless firefly 197 luciferase reporter vector, pGL3-Basic (Promega, Madison, WI, USA) generated constructs 198 pGL3-1063_-31 and pGL3-1063_-31 c.-98 C>A. Subsequently, a 4kb fragment, and a series of 199 nested deletions ranging from $2 \mathrm{~kb}$ to $44 \mathrm{bp}$ were cloned into pGL3-Basic with the same 200 restriction sites added and used for subsequent digest, to identify the minimal promoter necessary 201 and sufficient for transcription. pGL3-Basic lacks a eukaryotic promoter sequence upstream of 202 the reporter luciferase gene, and expression of luciferase in transfected cells depends on a 203 functional promoter sequence to be inserted upstream of the $l u c+$ gene. pGL3-Basic itself served 204 as a negative control. A schematic of all inserts assayed is provided in Figure 1A. All constructs 
205 generated were Sanger sequenced to ensure fidelity. Primer sequences for the generation of 206 plasmid inserts are available upon request.

207 Luciferase Assay

208 HEK293T cells cultured with standard reagents and conditions were seeded in 24 well plates and 209 transfected at 70\% confluency with polyethylenimine (PEI). Each well was transfected with a 210 total of $650 \mathrm{ng}$ of plasmid DNA, including 600ng of pGL3 construct and 50ng of the internal 211 control pRL-CMV, a cytomegalovirus promoter driven Renilla luciferase reporter vector. 212 Briefly, plasmid DNA was diluted into $25 \mu \mathrm{L}$ of PBS, and mixed by vortexing with $1 \mu \mathrm{L}$ of $2131 \mathrm{mg} / \mathrm{mL}$ branched PEI (Sigma-Aldrich, St. Louis, MO, USA) in 25 $\mu \mathrm{L}$ PBS. After a 15 minute 214 incubation, $50 \mu \mathrm{L}$ of the reaction was added dropwise to each well. Forty-eight hours after 215 transfection, luciferase activity was assayed using the Dual-Luciferase Reporter Assay kit and 216 measured with the Glomax Explorer (both Promega, Madison, WI, USA). Relative luciferase 217 activity was obtained by dividing the relative light units (RLU) produced by the firefly luciferase 218 pGL3 construct by the RLUs produced by Renilla luciferase control reaction. Light generated by 219 the reaction can be correlated with the amount of luciferase protein produced which in turn is 220 proportional to promoter activity driving the gene's expression. Values from an $\mathrm{n}=6$ were 221 averaged and normalized to that of the reference construct, pGL3-1093_-31 to obtain a relative 222 measure of activity.

223 Statistical analysis

224 All data were expressed as mean \pm SD. Data from the luciferase assay represents two 225 independent experiments with triplicate measurements. Differences between groups were 226 examined for statistical significance using Student's $t$-test. A $P$-value $<0.01$ denoted the presence 227 of a statistically significant difference. 
Promoter mutation causing choroideremia

228

229 Results

230

231 Clinical Characterization of CHM

232 Family 1

233 We investigated a progressive retinal degeneration in a Caucasian family of American origin 234 (C127). Remarkably, the proband 5116 was the offspring of a consanguineous union between 235 second cousins, with an affected father and carrier mother. To our knowledge, this has never 236 been reported with choroideremia, and it necessitated a thorough and accurate diagnosis of X237 linked CHM and exclusion of an autosomal recessive, or even an unusual male-male 238 transmission as cause for the disease. An investigation of the extended family for which the 239 pedigree is reported in Figure 2A however, clearly demonstrated the X-linked inheritance of the 240 disease.

241 Patient 5116 was initially seen at age 56 years, when he was referred by a retinal specialist with a 242 diagnosis of choroideremia. At his most recent examination, age 76, best corrected visual acuity 243 (BCVA) was measured as 6/19 OD and 6/15 OS. Visual fields were reduced to less than 5 244 degrees. The full-field ERG showed non-detectable dark adapted rod-driven responses as well as 245 non-detectable cone responses to a $30 \mathrm{~Hz}$ flicker stimulus. OCT imaging indicated loss of the 246 photoreceptor layer across the periphery of the fundus with only a small island of RPE remaining 247 in the macula. Posterior segment examination showed a hypo-pigmented fundus with significant 248 atrophy of the RPE and choroid with areas of bare sclera (Figure 3). These findings are 249 consistent with an advanced state of choroideremia. 
250 The proband's daughter 5113 was examined at 33 years of age and did not report any vision 251 difficulties. BCVA when examined was 6/6, both eyes. Full-field ERG testing of the left eye 252 showed dark adapted rod-driven responses were reduced by $50 \%$ in amplitude $(42.5 \mu \mathrm{V})$ and a 253 normal b-wave implicit time $(81.6 \mathrm{msec})$. Light-adapted cone-driven responses to a $30 \mathrm{~Hz}$ flicker 254 stimulus were reduced by $50 \%$ in amplitude $(27.6 \mu \mathrm{V})$ and borderline reduced in implicit time 255 (25.6 msec). These findings were consistent with a classic carrier state of choroideremia.

256 The proband's father and one brother 5149 were reported to have been previously diagnosed 257 with choroideremia, while a second brother 5147 was reported to be unaffected. Findings are 258 summarized in Table 1.

259 Family 2:

260 Family 2 (GC406) was a Caucasian family of British origin with a history of choroideremia. 261 Proband 111 was first examined at age 13, displaying a reduced but not delayed full field ERG 262 with flicker and bright flash responses both within normal limits. He remained asymptomatic 263 until age 32. Confrontational visual field testing at age 35 showed bilateral infratemporal 264 scotoma, and BCVA was $6 / 5$ in both eyes. He is considered symptomatically mild with retained 265 central macular structure.

266 Of his two maternal uncles, 151 was diagnosed at age 12. Fundus abnormalities typical of 267 choroideremia were noted. 161 was diagnosed at age 8 , and upon examination at age 37 268 exhibited moderately constricted visual fields particularly in the superior field bilaterally. BCVA 269 was reduced to count fingers vision OD and 6/36 OS. Fundus examination was consistent with a 270 clinical diagnosis of choroideremia. The pedigree is reported in Figure 2B, and clinical findings 271 summarized in Table 1. 
Promoter mutation causing choroideremia

\section{Genetic Analysis}

$274 \quad$ Family 1 (C127)

275 Genetic analysis of proband 5116 did not reveal any pathogenic mutation in the coding sequence 276 of the $C H M$ gene or splice site boundaries. Yet at position c.-98 relative to the translation start 277 site, a hemizygous C $>$ A transversion was detected (hg38, chrX:g.86047629G > T NM_000390.3, 278 c. $-98 \mathrm{C}>\mathrm{A})$. The variant was not listed in the latest release of dbSNP 279 [http://www.ncbi.nlm.nih.gov/SNP/; accessed Feb 2017] (Sherry et al., 2001). The proband's 280 affected brother (5149) was also found to harbour the variant while it was absent in the 281 unaffected brother (5147) available for testing. The obligate carrier status was confirmed in the 282 proband's daughter (5113). The location of the variant strongly suggested a regulatory mutation, 283 as evaluation of entries in the HGMD reveals that most promoter mutations are located between $284+50$ and -500 from the TSS of a gene (Stenson et al., 2014).

285 Family 2 (GC406):

286 Prior genetic analysis of the coding exons of the CHM gene did not reveal a pathogenic 287 mutation. Whole genome sequencing was performed on proband 111 and his parents 190 and 288 192, as part of the 100,000 Genomes Project. After variant filtering, no causative rare coding 289 variants were identified in any retinal disease gene.

290 In light of the clinical diagnosis of choroideremia in the family, the complete $C H M$ gene was 291 interrogated for rare variants $(\leq 0.001$ MAF in 1 Kgenome project and internal cohort of over 2922000 whole genome sequencing samples) hemizygous in the proband and carried by his mother. 293 One such variant was identified, the transition, c.-98C $>$ T (hg38, chrX:g.86047629G $>$ A 294 NM_000390.3, c.-98C>T). The variant was confirmed in the two affected maternal uncles 151 295 and 161 by direct Sanger sequencing. 


\section{Molecular diagnosis of choroideremia in 5116}

298 Immunoblot analysis of protein harvested from a cell line derived from patient 5116 failed to 299 detect the CHM gene product REP-1, providing conclusive confirmation of the clinical diagnosis 300 of choroideremia (Figure 4A). To provide evidence for a regulatory mutation and subsequently 301 reduced transcription, we intended to compare the level of expression between normal and 302 patient samples through qPCR. Endpoint PCR from a cDNA template; however, failed to 303 amplify the 15 exon, 2200 base pair transcript, indicating it was absent or present at a level 304 below the detection threshold, and thus the quantitative assay was not performed. To largely rule 305 out the possibility of a splice defect, we also attempted to amplify a minimal portion of the 306 transcript, a 93 base pair fragment from the 5'-UTR to a region spanning the boundary of exon 1 307 and 2, and found the patient's cells lacked even this short fragment (Figure 4B). A control 308 housekeeping gene was nevertheless readily detected and both partial and full length transcripts 309 were amplified from normal cDNA.

311 Effect of the c.-98C $>A$ and c.-98C $>$ T mutations on transcription of CHM

312 As a starting point, an approximately $1 \mathrm{~kb}$ fragment upstream of the TSS was assayed for ability 313 to drive gene expression, since the majority of elements necessary for transcription are expected 314 to be found within this region (Rockman and Wray, 2002). Comparing the robust luciferase 315 activity produced by cells transfected with this wild type construct pGL3-1093_-31, to that of 316 pGL3-1093_-31 c.-98C $>$ A and c. $-98 \mathrm{C}>\mathrm{T}$, we observed complete abrogation of promoter activity 317 (Figure 1B) in the mutants. The drop from $100 \pm 9.5$ to $2.0 \pm 0.3$ and $1.3 \pm 0.4$ respectively, as 318 measured in normalized relative light units $(\mathrm{RLU}) \pm 1$ standard deviation is even significantly 
319 lower than that of the negative control pGL3-basic which does not contain a promoter sequence,

320 reading at $5.8 \pm 0.9$. This startling observation strongly suggested that the mutation spans an

321 element essential to promoter activity.

322 Based on unpublished reports suggesting other activating elements may exist up to $2.8 \mathrm{~kb}$ 323 upstream of the TSS (Kaiser NW, et al. IOVS 2004;45:ARVO E-Abstract 2451), we additionally 324 compared the activity of two larger constructs approximately $4 \mathrm{~kb}$ and $2 \mathrm{~kb}$ long. Typically, 325 regulatory regions upstream of core promoter sequences contain multiple TF specific binding 326 motifs, where several copies of the same factor or cooperation between different factors serve to 327 synergistically stimulate transcriptional activity of a given gene (Maston et al., 2006). Yet in the 328 case of $C H M$, the longer sequences did not stimulate additional luciferase expression. pGL3329 3983_-31 and pGL3-2027_-31 did not significantly differ from pGL3-1093_-31, producing 83.7 $330 \pm 4.9$ and $96.2 \pm 7.6$ RLUs respectively. The somewhat diminished expression as compared to 331 the reference $1 \mathrm{~kb}$ construct may be attributed to a lower molar amount of the larger plasmids 332 being delivered during transfection.

334 Characterization of CHM promoter

335 To further characterize the promoter, we proceeded to assay progressively shorter constructs, 336 deleting sequences from the $-5^{\prime}$ and $-3^{\prime}$ of the $1 \mathrm{~kb}$ construct to define its boundaries (Figure 1A). 337 Working from the 5' end, the approximately 400, 300, and 100 base pair constructs pGL3-437_338 31, pGL3-346_-31, pGL3-119_-31 did not significantly differ from the $1 \mathrm{~kb}$ containing reference 339 plasmid pGL3-1093_-31, reading at 100.2 $\pm 11.4,96.1 \pm 10.6$, and 97.7 \pm 8.1 RLU respectively. 340 Yet upon deleting a further 11 base pairs, we observed a significant drop to $39.8 \pm 7.6$ RLU with 341 pGL3-108_-31. Having delineated the -5 ' boundary of the promoter as extending to no further 
342 than nucleotide c.-119, we subsequently tested pGL3-119_-76 bearing a deletion from the -3 ' 343 end, and found no significant difference at $93.4 \pm 14$ RLU. It was the final construct, pGL3344 119_-82, which yielded significantly lower activity producing only $27.4 \pm 4$ RLU. Though 345 further characterization of the promoter region down to the single nucleotide level could be 346 performed, we have defined the borders of the promoter to within the region of c.-119 to c.-76.

347 This short 44 base pair DNA region upstream of the $C H M$ coding sequence is able to stimulate 348 transcription in an in-vitro assay at a level that is not significantly different from that of a $1 \mathrm{~kb}$, or 349 even a $4 \mathrm{~kb}$ fragment and can be understood to contain essential cis-acting elements that 350 positively regulate $C H M$ expression.

352 Discussion

353 To date, all genetic defects causing choroideremia, which include all major mutation classes, 354 have been observed in exons, introns or intron/exon boundaries of the $C H M$ gene (Fokkema et 355 al., 2011). No mutations have been reported in the promoter region of $C H M$, and the regulation 356 of the gene has remained essentially unexplored. In this study, we present the first report of 357 promoter mutations, c. $-98 \mathrm{C}>\mathrm{A}$ and c. $-98 \mathrm{C}>\mathrm{T}$, causing choroideremia. The critical role of this 358 residue for gene expression is highlighted by the complete abrogation of reporter activity in a 359 promoter assay when the nucleotide is mutated. Though we demonstrate the first examples in a 360 novel mutation class, it is worthwhile noting that the phenotypes observed in affected individuals 361 were typical of choroideremia. In family 1, the genotype c.-98C $>\mathrm{A}$ in proband 5116 resulted in 362 severe retinopathy, while characteristic milder signs were found in the carrier female 5113.

363 Males from family 2 bearing the c.-98C $>$ T mutation manifested with chorioretinal disease, but 364 with varying degrees of severity; proband 111 remained symptomatically mild while in his 
365 maternal uncles 151 and 161, disease progression was more typical. CHM is known to exhibit a

366 wide interfamilial, and also intrafamilial variability (Moosajee et al., 2014). As the mutations c.-

$36798 \mathrm{C}>\mathrm{A}$ and c. $-98 \mathrm{C}>\mathrm{T}$ appear to completely abolish transcription, producing no detectable levels

368 of $C H M$ mRNA and REP-1 protein in patient cells, it is not surprising for the disease to present

369 in a classical manner consistent with phenotypes observed for the loss of function mutations.

370 Whole genome sequencing in the parent-offspring trio of family 2 allowed the exclusion of a

371 deep intronic CHM mutation, as well as mono or biallelic mutations in any other retinal

372 dystrophy gene as a cause of the observed symptoms. We therefore conclude that the two

373 mutations at residue c.-98 impair $C H M$ transcription enough to result in choroideremia. For the

374 several gene therapy trials currently underway employing a "gene replacement" strategy

375 (Dimopoulos et al., 2015), individuals bearing these genotypes would be suitable candidates. The

376 two variants have been submitted and published in the LOVD Retinal and Hearing Impairment

377 Genetic Mutation Database (Fokkema et al., 2011).

378

379 Having identified mutations that evidently abolished transcription, we interpreted their location 380 to span a region crucial for gene expression, and set out to define the boundaries of the gene's 381 promoter. Surprisingly, after analyzing fragments as long as $4 \mathrm{~kb}$, we found a short 44 base pair 382 DNA fragment to be wholly responsible for driving expression. The region c.-119 to c.-76 383 comprises the entirety of the $C H M$ proximal promoter, and is able to drive robust transcription in 384 an in-vitro luciferase assay. The region implicated amounts to less than $3 \%$ of the length of the 385 CHM coding sequence where the large majority of reported causative mutations have been found 386 (Fokkema et al., 2011). A recent investigation of a large disease cohort (74), found causative 387 mutations in the gene in 94\% of cases previously diagnosed with CHM (Simunovic et al., 2016). 
388 The remaining $6 \%$ can be understood to be comprised of regulatory mutations, incorrect 389 diagnoses or deep intronic variant causing cryptic splicing (Carss et al., 2017). Promoter defects 390 are therefore expected to be responsible for a small minority of choroideremia cases. The 391 identified region; however, becomes an obvious area for examination in patients in whom no 392 coding sequence mutation is found.

394 A promoter or regulatory mutation can be expected to either increase or decrease transcriptional 395 activity mediated by the altered binding capacity of trans-acting protein factors specific to a 396 DNA sequence in the promoter region. In this case, the interaction appears to be entirely 397 disrupted based on the null expression of a reporter driven by mutated sequence in our luciferase 398 assay. Impaired transcription due to a 1-bp mutation in a promoter region is unusual, but has 399 been reported previously as in the case of single base mutations stimulating additional 400 transcriptional activity at the OVOL2 promoter causing autosomal-dominant corneal endothelial 401 dystrophies (Davidson et al., 2016). On the other hand, decreased transactivation of NMNAT1 402 due to a single nucleotide change in the promoter was found to cause Leber congenital amaurosis 403 (Coppieters et al., 2015).

404

405 While we delineate the CHM promoter boundary to this small area upstream of the gene, we 406 cannot exclude the possibility of distant enhancer, repressor, or intronic elements also 407 contributing to regulation; our findings suggest the sequence between c.-119 and c.-76 is 408 essential, but not necessarily sufficient for transcription. The wider upstream sequence of $C H M$ 409 lacks the consensus sequences often found in RNA polymerase II promoters, such as CAAT and 410 TATA boxes; as well it is neither $\mathrm{GC}$ rich nor associated with any $\mathrm{CpG}$ islands. Bioinformatic 
411 analysis with MotifMap, a dataset of computationally predicted transcription factor binding sites

412 based on binding motifs [http://motifmap.ics.uci.edu/] (Daily et al., 2011) identifies a putative

413 binding motif for the transcription factor zinc finger protein 143 (ZNF143) contained within the

414 region of the now revealed $C H M$ promoter. ZNF143 participates in the regulation of RNA pol II

415 and III mediated transcription of protein coding, non-coding, and small nuclear RNA (Schaub et

416 al., 1997; Myslinski et al., 1998) and was initially connected with the binding motif

417 TTCCCATTATGCACCGCG (SBS1) (Myslinski et al., 2006). Genome-wide studies revealed

418 the binding site for the factor to be frequently found with an adjacent 5' accessory sequence,

419 forming the ACTACAATTCCCATTATGCACCGCG (SBS2) motif. SBS2 is comprised of both

420 a THAP domain-containing protein 11 (THAP11) and ZNF143 binding site, with the factors

421 believed to act in a competitive manner (Ngondo-Mbongo et al., 2013). The recruitment of

422 THAP11 to its canonical binding site ACTAYRNNNCCCR is most frequently associated with

423 up-regulation genes essential to protein biosynthesis and energy production (Dejosez et al.,

424 2010). More recently, ZNF143 was suggested to cooperatively occupy SBS2 sites with THAP11

425 and a third factor, the scaffold protein host cell factor 1 (HCFC1) in-vivo (Vinckevicius et al.,

426 2015). SBS2 is closely matched by the sequence found at position c.-108 to c.-84 upstream of

427 CHM, ACTACAACACCCAGAATGCACTGTT. Notably, ZNF143's binding at promoters was

428 recently implicated in chromatin looping with distal regulatory elements (Bailey et al., 2015),

429 suggesting the involvement of yet other factors in the total regulation of expression of CHM.

430

431 Of the three transcription factors implicated above, the binding of at least ZNF143 is supported

432 by publicly available chromatin immunoprecipitation sequencing data released as part of the

433 ENCODE project [https://genome.ucsc.edu/ENCODE/] (ENCODE Project Consortium, 2012). 
434 In all cell types assayed: lymphoblastoid, HeLa and K562 cell lines, as well as embryonic stem 435 cells, the promoter region of $C H M$ interacted with ZNF143, for which a consensus binding 436 sequence GAACTACAATTCCCAGAAGGC, again is closely matched by 437 GAACTACAACACCCAGAATGC found between position c.-110 toc.-91 relative to the A of 438 the CHM start codon. The position-weight matrix for ZNF143 (Figure 5) establishes the relative 439 frequency of the base $\mathrm{C}$ at position c. -98 to be $100 \%$, supporting the importance of the residue 440 and the resulting pathogenesis when mutated. Furthermore, surveying the UCSC genome 441 browser (http://genome.ucsc.edu/) multiz alignment of 100 vertabrate genomes tract, the region 442 c.-119 to c.-76 shows a high degree of conservation among mammals, and absolute conservation 443 of residue $\mathrm{C}$ at positions corresponding to c.-98 pointing to an important biological role for the 444 sequence (Kuhn et al., 2007; Blanchette et al., 2004). Representatives of birds and amphibian 445 classes, however, lack homology in the region, in while in fish a corresponding region is absent 446 altogether. The promoter, therefore, cannot be considered an ultra-conserved non-coding element 447 (Dimitrieva and Bucher, 2013). Several alignments are listed in Table 2.

449 Studies of $C H M$ mRNA and protein localization have found a broad expression profile for both.

450 In mice, evidence of transcription was found in multiple cell types and in every major layer of 451 retina (Keiser et al., 2005), while immunolabeling of primate retina showed REP-1 localized to 452 both rod and cones (Dimopoulos et al., 2015). Studies in human and primate retina found that 453 mRNA levels did not correspond to the pattern of disease expression; little CHM was detected in 454 the RPE and choroid, and there were no marked regional differences in the concentration of 455 CHM mRNA apparent with foveal versus mid-peripheral total RNA despite affected males 456 typically exhibiting a preservation of central vision until late in the disease (Bernstein and Wong, 
457 1998). Additionally, REP-1 can be readily detected in human fibroblasts or peripheral blood 458 mononuclear cells (Furgoch et al., 2014; MacDonald et al., 1998). Taken together with 459 ZNF143's characterization as one of the most common and ubiquitously expressed TFs 460 (Myslinski et al., 1998) a picture emerges of widespread and non-specific transcription of CHM, 461 despite choroideremia's manifestation as an ocular disease. Indeed, patients' apparent lack of 462 systemic symptoms can be understood to result not from tissue specific expression of REP-1, but 463 from the differing affinities of REP-1 and REP-2 for target Rabs, which may themselves be 464 differentially expressed or possess tissue or cell specific activity. Investigators have implicated 465 Rab27 (Seabra et al., 1995) and Rab38 (Kohnke et al., 2013) as possible contributors.

467 The study presented here also poses interesting questions, such as whether mutations of other 468 residues less critical to transactivator binding in the $C H M$ promoter, that diminish, but not 469 completely abolish mRNA expression, can result in a milder phenotype, or a different rate of 470 progression of choroideremia. Currently, the dbSNP database lists no known SNPs between c.471119 to c.-76 (Sherry et al., 2001). Having shown the CHM region responsible for regulation of its 472 expression, described for the first time the features of its promoter, and extended the inventory of 473 molecular changes causing choroideremia, the findings are of clinical and diagnostic interest and 474 present an obvious area of examination for patients with CHM in whom no coding sequence 475 mutation has been found. Further elucidating the roles of ZNF143, THAP11, HCFC1 or other 476 distal factors will prove an important step toward understanding the complete picture of $C H M$ 's 477 regulation. 
480 The authors have no proprietary or commercial interest in any materials discussed in this article.

481 Support by Canadian Institutes of Health Research, Emerging Team Grant: 119190, Foundation

482 Fighting Blindness, Canada, Choroideremia Research Foundation Canada, Inc., and Alberta

483 Innovates-Health Solutions 201201139 is acknowledged (IM).

484 This project was also supported by The National Institute for Health Research (NIHR) and 485 Biomedical Research Centre (BRC) at Moorfields Eye Hospital and the UCL Institute of 486 Ophthalmology (GA, AW).

487 This research was made possible through access to the data and findings generated by the 488 100,000 Genomes Project. The 100,000 Genomes Project is managed by Genomics England 489 Limited (a wholly owned company of the Department of Health). The 100,000 Genomes Project 490 is funded by the NIHR and NHS England. The Wellcome Trust, Cancer Research UK and the 491 Medical Research Council have also funded research infrastructure.

492

493 Support by the National Eye Institute grant EY-09076, and Foundation Fighting Blindness is also 494 acknowledged (DB)

495

496

497

498

$499 \quad$ Figure Legends

500 Figure 1. Functional Analysis of the $C H M$ Promoter. 
501 (A) The constructs portrayed on the left were inserted upstream of the luciferase gene in pGL3-

502 basic. Nucleotide +1 represents the translation start site. The promoterless pGL3-basic served as

503 a negative control. (B) The mutations c. $-98 \mathrm{C}>\mathrm{A}$, and c. $-98 \mathrm{C}>\mathrm{T}$ abolish transcription, while the

504 minimal construct c.-119_-76 is sufficient for robust expression of the reporter gene not

505 significantly different from even that of the nearly $4 \mathrm{~kb}$ construct. All constructs were transiently

506 transfected into HEK293T cells. A dual-luciferase reporter assay was used to assess the potential

507 promoter activity of various sized inserts and the c.-98 mutants. Promoter activity is shown as a

508 ratio of firefly luciferase over Renilla luciferase present on the transfection control plasmid pRL-

$509 \mathrm{CMV}$ to account for inter-well variation. Activity is normalized to that of the reference construct

510 pGL3-1093_-31 which is artificially set to equal 100. Activity significantly different $(\mathrm{P}<0.01)$

511 from the reference construct is denoted by an asterisk. Error bars represent $\pm 1 \mathrm{SD}$.

512 Figure 2. Pedigree Structure of Affected Families with CHM Promoter Mutations

513 (A) Family1. The parents of proband 5116 were second cousins, sharing a set of great grand-

514 parents. The inheritance pattern mimics male-male transmission, but is nevertheless consistent

515 with X-linked inheritance upon examination of the wider family pedigree. (B) Family 2. The

516 pedigree showing three generations affected by choroideremia examined in this study.

517 Inheritance follows an X-linked pattern.

518 Figure 3. Retinal features in patient 5116.

519 Legend: Left column (OD), right column (OS). (A) Fundus photographs of the proband taken at 520 age 76 showing typical choroideremia changes, with atrophy of the choroid and RPE. A small

521 island of preserved RPE remains in the central macula, surrounded by atrophic peripheral areas

522 of apparent bare sclera. (B)(C) Fundus autofluorescence image demonstrating areas of residual

523 RPE (L) and the corresponding OCT image (R). Preserved retinal areas with normal 
524 autofluorescence exhibit thicker choroid and preserved retinal lamination. An outer retinal 525 tubulation is seen in the right fundus.

526 Figure 4. Molecular confirmation of choroideremia in patient 5116.

527 (A) Patient 5116 lacks REP-1. Western blot results show the absence of a $\sim 100 \mathrm{kDa}$ band 528 corresponding to REP-1 in a lymphoblastoid cell line derived from the patient (lane 2), which is 529 present in a normal control (lane 1). A $\beta$-actin antibody was used as a loading control to ensure 530 an adequate protein sample in each lane, with the $42 \mathrm{kDa}$ band present in both samples.

531 (B) Patient 5116 lacks CHM mRNA. cDNA synthesized from the mRNA harvested from a 532 patient generated lymphoblastoid cell line was used as template for PCR. Lanes 2 and 3 show a $533475 \mathrm{bp}$ band resulting from the amplification of the GAPDH control housekeeping gene from 5345116 and a normal control, indicating cDNA of adequate quality. Lanes 6 and 9 demonstrate an 535 absence of amplification from the patient's cDNA of both partial and full length coding 536 sequence, respectively, as compared to PCR products sized 93 and 2200 base pairs amplified 537 from normal cDNA observed in lanes 5 and 10.

538 Figure 5. Consensus binding sequences for transcription factor ZNF143.

539 (A) Partial map of the human $C H M$ gene; arrow indicates transcription start site. (B) Expanded 540 sequence of the minimal $C H M$ promoter from c.-119 to c.-76, as identified through the analysis 541 of progressive deletion constructs. Position c.-98 is marked with an asterisk. (C) Sequence logo 542 derived from publically available ChIP-seq data released as part of the ENCODE project, with 543 the position weighted matrix below (ENCODE Project Consortium, 2012). An invariant $\mathrm{C}$ is 544 found at position corresponding to c.-98 of $\mathrm{CHM}$.

546 Table 1. Clinical data of genotyped individuals 
Promoter mutation causing choroideremia

547 Table 2. Multiz alignment of the promoter region of 15 CHM orthologs

548

549 References

550 Anderson MA, Gusella JF. 1984. Use of cyclosporin A in establishing epstein-barr virus-

551 transformed human lymphoblastoid cell lines. In Vitro 20:856-858.

552 Bailey SD, Zhang X, Desai K, Aid M, Corradin O, Cowper-Sal Lari R, Akhtar-Zaidi B, Scacheri

553 PC, Haibe-Kains B, Lupien M. 2015. ZNF143 provides sequence specificity to secure chromatin

554 interactions at gene promoters. Nat Commun 2:6186.

555 Bernstein SL, Wong P. 1998. Regional expression of disease-related genes in human and 556 monkey retina. Mol Vis 4:24.

557 Blanchette M, Kent WJ, Riemer C, Elnitski L, Smit AF, Roskin KM, Baertsch R, Rosenbloom

558 K, Clawson H, Green ED, Haussler D, Miller W. 2004. Aligning multiple genomic sequences

559 with the threaded blockset aligner. Genome Res 14:708-715.

560 Carss KJ, Arno G, Erwood M, Stephens J, Sanchis-Juan A, Hull S, Megy K, Grozeva D,

561 Dewhurst E, Malka S, Plagnol V, Penkett C, Stirrups K, Rizzo R, Wright G, Josifova D, Bitner-

562 Glindzicz M, Scott RH, Clement E, Allen L, Armstrong R, Brady AF, Carmichael J, Chitre M,

563 Henderson RH, Hurst J, MacLaren RE, Murphy E, Paterson J, Rosser E, Thompson DA,

564 Wakeling E, Ouwehand WH, Michaelides M, Moore AT, NIHR-BioResource Rare Diseases

565 Consortium, Webster AR, Raymond FL. 2017. Comprehensive rare variant analysis via whole-

566 genome sequencing to determine the molecular pathology of inherited retinal disease. Am J Hum

567 Genet 100:75-90. 
568 Chi JY, MacDonald IM, Hume S. 2013. Copy number variant analysis in CHM to detect

569 duplications underlying choroideremia. Ophthalmic Genet 34:229-233.

570 Coppieters F, Todeschini AL, Fujimaki T, Baert A, De Bruyne M, Van Cauwenbergh C, Verdin

571 H, Bauwens M, Ongenaert M, Kondo M, Meire F, Murakami A, Veitia RA, Leroy BP, De Baere

572 E. 2015. Hidden genetic variation in LCA9-associated congenital blindness explained by 5'UTR

573 mutations and copy-number variations of NMNAT1. Hum Mutat 36:1188-1196.

574 Coussa RG, Traboulsi EI. 2012. Choroideremia: A review of general findings and pathogenesis.

575 Ophthalmic Genet 33:57-65.

576 Cremers FP, Armstrong SA, Seabra MC, Brown MS, Goldstein JL. 1994. REP-2, a rab escort

577 protein encoded by the choroideremia-like gene. J Biol Chem 269:2111-2117.

578 Cremers FP, van de Pol DJ, van Kerkhoff LP, Wieringa B, Ropers HH. 1990. Cloning of a gene

579 that is rearranged in patients with choroideraemia. Nature 347:674-677.

580 Daily K, Patel VR, Rigor P, Xie X, Baldi P. 2011. MotifMap: Integrative genome-wide maps of 581 regulatory motif sites for model species. BMC Bioinformatics 12:495-2105-12-495.

582 Davidson AE, Liskova P, Evans CJ, Dudakova L, Noskova L, Pontikos N, Hartmannova H, 583 Hodanova K, Stranecky V, Kozmik Z, Levis HJ, Idigo N, Sasai N, Maher GJ, Bellingham J, Veli 584 N, Ebenezer ND, Cheetham ME, Daniels JT, Thaung CM, Jirsova K, Plagnol V, Filipec M, 585 Kmoch S, Tuft SJ, Hardcastle AJ. 2016. Autosomal-dominant corneal endothelial dystrophies 586 CHED1 and PPCD1 are allelic disorders caused by non-coding mutations in the promoter of 587 OVOL2. Am J Hum Genet 98:75-89. 
Promoter mutation causing choroideremia

588 de Vooght KM, van Wijk R, van Solinge WW. 2009. Management of gene promoter mutations

589 in molecular diagnostics. Clin Chem 55:698-708.

590 Dejosez M, Levine SS, Frampton GM, Whyte WA, Stratton SA, Barton MC, Gunaratne PH, 591 Young RA, Zwaka TP. 2010. Ronin/hcf-1 binds to a hyperconserved enhancer element and

592 regulates genes involved in the growth of embryonic stem cells. Genes Dev 24:1479-1484.

593 Dimitrieva S, Bucher P. 2013. UCNEbase--a database of ultraconserved non-coding elements 594 and genomic regulatory blocks. Nucleic Acids Res 41:D101-9.

595 Dimopoulos IS, Chan S, MacLaren RE, MacDonald IM. 2015. Pathogenic mechanisms and the 596 prospect of gene therapy for choroideremia. Expert Opin Orphan Drugs 3:787-798.

597 ENCODE Project Consortium. 2012. An integrated encyclopedia of DNA elements in the human 598 genome. Nature 489:57-74.

599 Esposito G, De Falco F, Tinto N, Testa F, Vitagliano L, Tandurella IC, Iannone L, Rossi S, 600 Rinaldi E, Simonelli F, Zagari A, Salvatore F. 2011. Comprehensive mutation analysis (20

601 families) of the choroideremia gene reveals a missense variant that prevents the binding of REP1 602 with rab geranylgeranyl transferase. Hum Mutat 32:1460-1469.

603 Fokkema IF, Taschner PE, Schaafsma GC, Celli J, Laros JF, den Dunnen JT. 2011. LOVD v.2.0:

604 The next generation in gene variant databases. Hum Mutat 32:557-563.

605 Freund PR, Sergeev YV, MacDonald IM. 2016. Analysis of a large choroideremia dataset does 606 not suggest a preference for inclusion of certain genotypes in future trials of gene therapy. Mol 607 Genet Genomic Med 4:344-358. 
608 Furgoch MJB, Mewes-Arès J, Radziwon A, MacDonald IM. 2014. Molecular genetic diagnostic 609 techniques in choroideremia. Mol Vision 20:535-544.

610 Karna J. 1986. Choroideremia. A clinical and genetic study of 84 finnish patients and 126 female 611 carriers. Acta Ophthalmol Suppl 176:1-68.

612 Keiser NW, Tang W, Wei Z, Bennett J. 2005. Spatial and temporal expression patterns of the 613 choroideremia gene in the mouse retina. Mol Vision 11:1052-1060.

614 Kohnke M, Delon C, Hastie ML, Nguyen UT, Wu YW, Waldmann H, Goody RS, Gorman JJ, 615 Alexandrov K. 2013. Rab GTPase prenylation hierarchy and its potential role in choroideremia 616 disease. PLoS One 8:e81758.

617 Kuhn RM, Karolchik D, Zweig AS, Trumbower H, Thomas DJ, Thakkapallayil A, Sugnet CW, 618 Stanke M, Smith KE, Siepel A, Rosenbloom KR, Rhead B, Raney BJ, Pohl A, Pedersen JS, Hsu 619 F, Hinrichs AS, Harte RA, Diekhans M, Clawson H, Bejerano G, Barber GP, Baertsch R, 620 Haussler D, Kent WJ. 2007. The UCSC genome browser database: Update 2007. Nucleic Acids 621 Res 35:D668-73.

622 MacDonald IM, Mah DY, Ho YK, Lewis RA, Seabra MC. 1998. A practical diagnostic test for 623 choroideremia. Ophthalmology 105:1637-1640.

624 Maston GA, Evans SK, Green MR. 2006. Transcriptional regulatory elements in the human 625 genome. Annu Rev Genomics Hum Genet 7:29-59.

626 McTaggart KE, Tran M, Mah DY, Lai SW, Nesslinger NJ, MacDonald IM. 2002. Mutational 627 analysis of patients with the diagnosis of choroideremia. Hum Mutat 20:189-196. 
Promoter mutation causing choroideremia

628 Moosajee M, Ramsden SC, Black GC, Seabra MC, Webster AR. 2014. Clinical utility gene card 629 for: Choroideremia. Eur J Hum Genet 22:10.1038/ejhg.2013.183. Epub 2013 Aug 21.

630 Myslinski E, Gerard MA, Krol A, Carbon P. 2006. A genome scale location analysis of human 631 staf/ZNF143-binding sites suggests a widespread role for human staf/ZNF143 in mammalian 632 promoters. J Biol Chem 281:39953-39962.

633 Myslinski E, Krol A, Carbon P. 1998. ZNF76 and ZNF143 are two human homologs of the 634 transcriptional activator staf. J Biol Chem 273:21998-22006.

635 Ngondo-Mbongo RP, Myslinski E, Aster JC, Carbon P. 2013. Modulation of gene expression via 636 overlapping binding sites exerted by ZNF143, Notch1 and THAP11. Nucleic Acids Res 41:40006374014.

638 Roberts MF, Fishman GA, Roberts DK, Heckenlively JR, Weleber RG, Anderson RJ, Grover S. 639 2002. Retrospective, longitudinal, and cross sectional study of visual acuity impairment in 640 choroideraemia. Br J Ophthalmol 86:658-662.

641 Rockman MV, Wray GA. 2002. Abundant raw material for cis-regulatory evolution in humans. 642 Mol Biol Evol 19:1991-2004.

643 Schaub M, Myslinski E, Schuster C, Krol A, Carbon P. 1997. Staf, a promiscuous activator for 644 enhanced transcription by RNA polymerases II and III. Embo J 16:173-181.

645 Seabra MC, Brown MS, Goldstein JL. 1993. Retinal degeneration in choroideremia: Deficiency 646 of rab geranylgeranyl transferase. Science 259:377-381. 
647 Seabra MC, Brown MS, Slaughter CA, Sudhof TC, Goldstein JL. 1992. Purification of

648 component A of rab geranylgeranyl transferase: Possible identity with the choroideremia gene 649 product. Cell 70:1049-1057.

650 Seabra MC, Ho YK, Anant JS. 1995. Deficient geranylgeranylation of ram/Rab27 in

651 choroideremia. J Biol Chem 270:24420-24427.

652 Sergeev YV, Smaoui N, Sui R, Stiles D, Gordiyenko N, Strunnikova N, MacDonald IM. 2009.

653 The functional effect of pathogenic mutations in rab escort protein 1. Mutat Res 665:44-50.

654 Sherry ST, Ward MH, Kholodov M, Baker J, Phan L, Smigielski EM, Sirotkin K. 2001. dbSNP:

655 The NCBI database of genetic variation. Nucleic Acids Res 29:308-311.

656 Simunovic MP, Jolly JK, Xue K, Edwards TL, Groppe M, Downes SM, MacLaren RE. 2016.

657 The spectrum of CHM gene mutations in choroideremia and their relationship to clinical

658 phenotype. Invest Ophthalmol Vis Sci 57:6033-6039.

659 Stenson PD, Mort M, Ball EV, Shaw K, Phillips A, Cooper DN. 2014. The human gene mutation

660 database: Building a comprehensive mutation repository for clinical and molecular genetics, 661 diagnostic testing and personalized genomic medicine. Hum Genet 133:1-9.

662 van Bokhoven H, van den Hurk JA, Bogerd L, Philippe C, Gilgenkrantz S, de Jong P, Ropers 663 HH, Cremers FP. 1994. Cloning and characterization of the human choroideremia gene. Hum 664 Mol Genet 3:1041-1046.

665 Van Den Hurk JAJM, Van De Pol DJR, Wissinger B, Van Driel MA, Hoefsloot LH, De Wijs IJ, 666 Van Den Born I, Heckenlively JR, Brunner HG, Zrenner E, Ropers H-, Cremers FPM. 2003. 
Promoter mutation causing choroideremia

667 Novel types of mutation in the choroideremia (CHM) gene: A full-length L1 insertion and an 668 intronic mutation activating a cryptic exon. Hum Genet 113:268-275.

669 Vinckevicius A, Parker JB, Chakravarti D. 2015. Genomic determinants of

670 THAP11/ZNF143/HCFC1 complex recruitment to chromatin. Mol Cell Biol 35:4135-4146.

671

John Wiley \& Sons, Inc. 


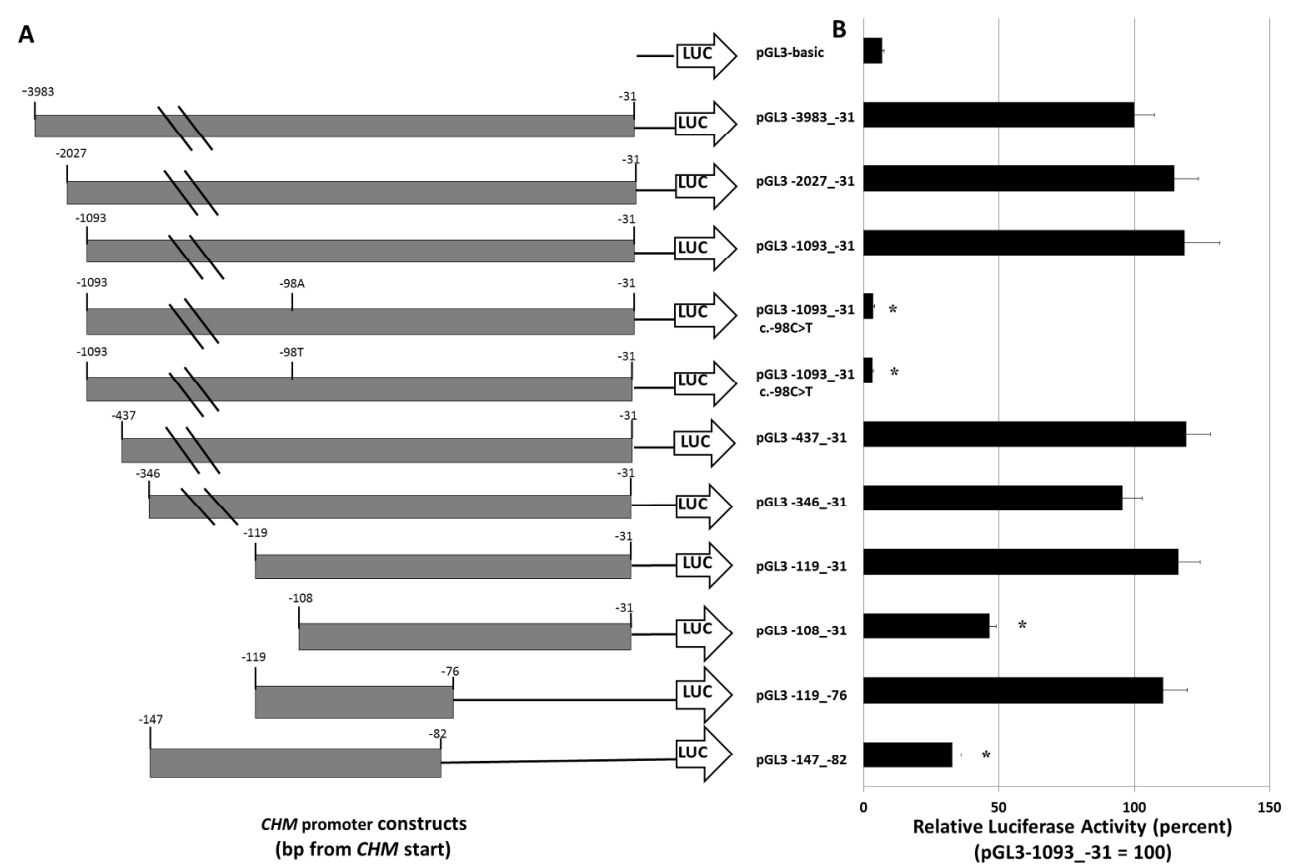

Figure 1. Functional Analysis of the CHM Promoter.

(A) The constructs portrayed on the left were inserted upstream of the luciferase gene in pGL3-basic. Nucleotide +1 represents the translation start site. The promoterless pGL3-basic served as a negative control. (B) The mutations c.-98C $>$ A, and c. $-98 \mathrm{C}>$ T abolish transcription, while the minimal construct c.119_-76 is sufficient for robust expression of the reporter gene not significantly different from even that of the nearly $4 \mathrm{~kb}$ construct. All constructs were transiently transfected into HEK293T cells. A dual-luciferase reporter assay was used to assess the potential promoter activity of various sized inserts and the c.-98 mutants. Promoter activity is shown as a ratio of firefly luciferase over Renilla luciferase present on the transfection control plasmid pRL-CMV to account for inter-well variation. Activity is normalized to that of the reference construct pGL3-1093_-31 which is artificially set to equal 100. Activity significantly different $(P<0.01)$ from the reference construct is denoted by an asterisk. Error bars represent \pm 1 SD. Figure 1 $609 \times 406 \mathrm{~mm}(96 \times 96 \mathrm{DPI})$ 


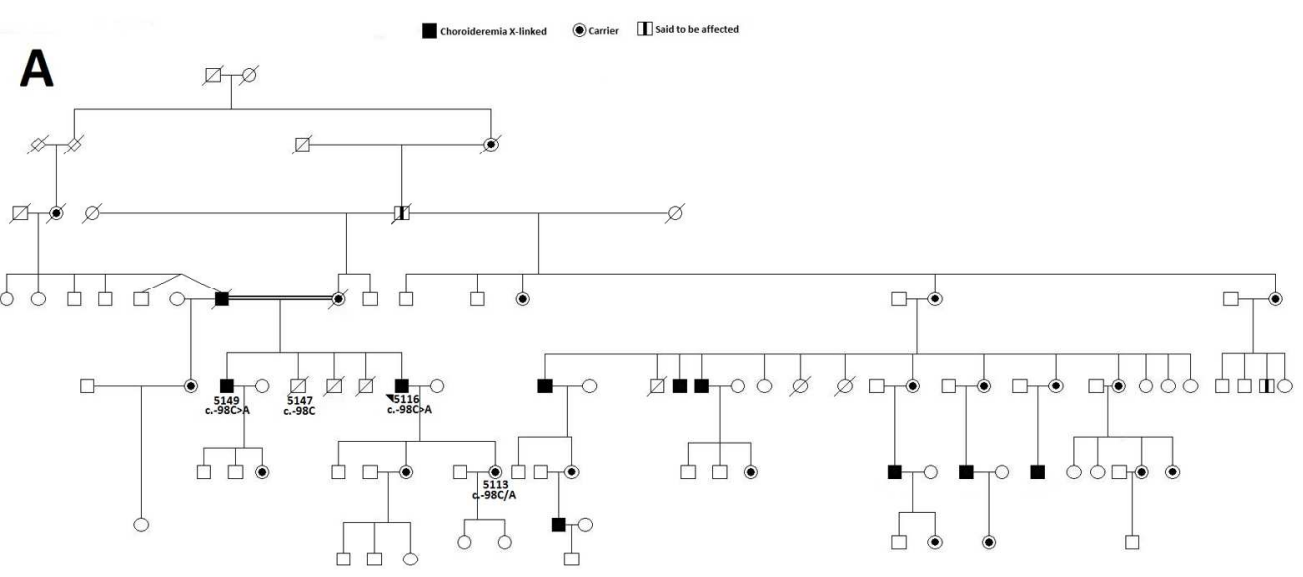

B

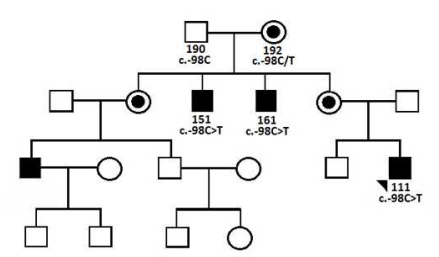

Figure 2. Pedigree Structure of Affected Families with CHM Promoter Mutations

(A) Family1. The parents of proband 5116 were second cousins, sharing a set of great grand-parents. The inheritance pattern mimics male-male transmission, but is nevertheless consistent with X-linked inheritance upon examination of the wider family pedigree. (B) Family 2 . The pedigree showing three generations affected by choroideremia examined in this study. Inheritance follows an X-linked pattern.

Figure 2

$478 \times 349 \mathrm{~mm}(96 \times 96 \mathrm{DPI})$

John Wiley \& Sons, Inc. 

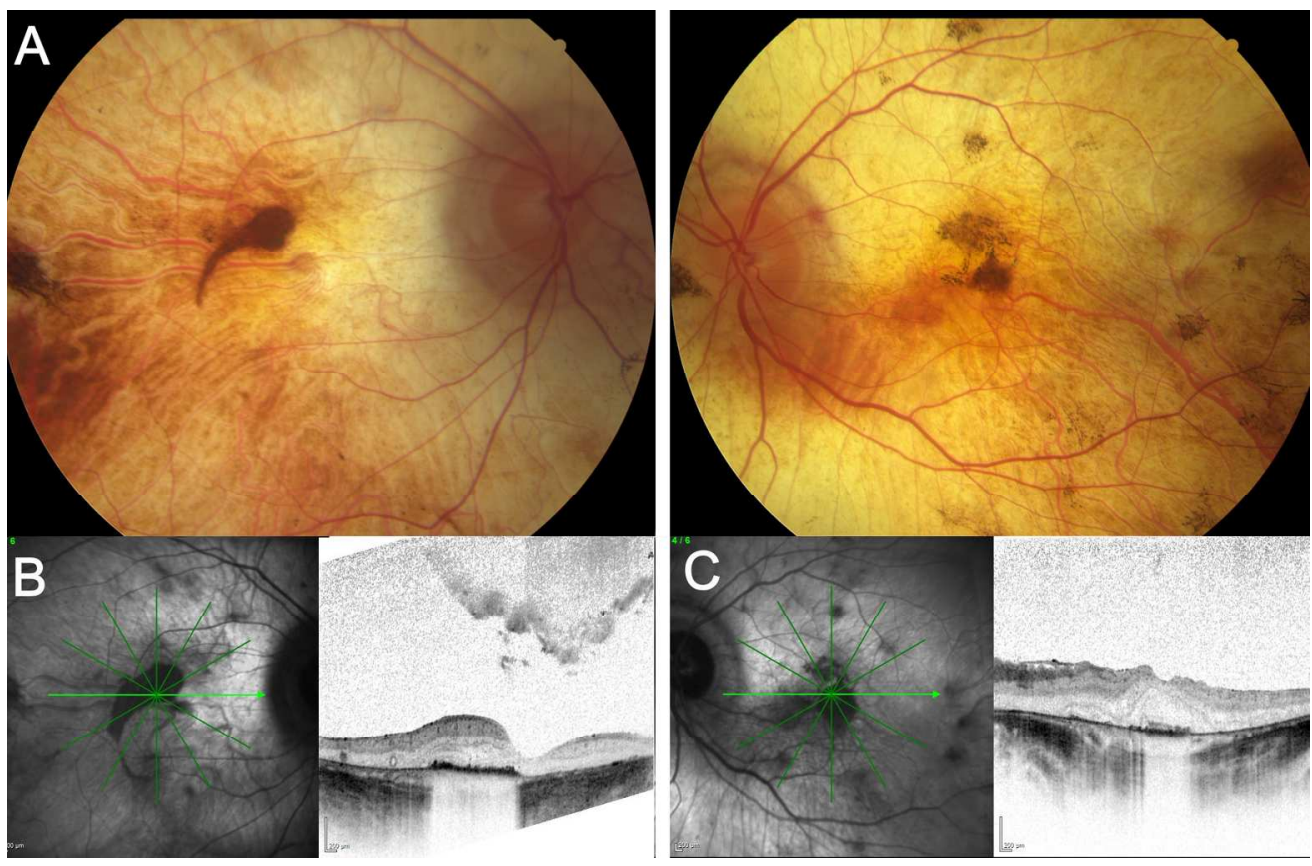

Figure 3. Retinal features in patient 5116.

Legend: Left column (OD), right column (OS). (A) Fundus photographs of the proband taken at age 76 showing typical choroideremia changes, with atrophy of the choroid and RPE. A small island of preserved RPE remains in the central macula, surrounded by atrophic peripheral areas of apparent bare sclera. (B)(C) Fundus autofluorescence image demonstrating areas of residual RPE $(L)$ and the corresponding OCT image

(R). Preserved retinal areas with normal autofluorescence exhibit thicker choroid and preserved retinal lamination. An outer retinal tubulation is seen in the right fundus.

Figure 3 

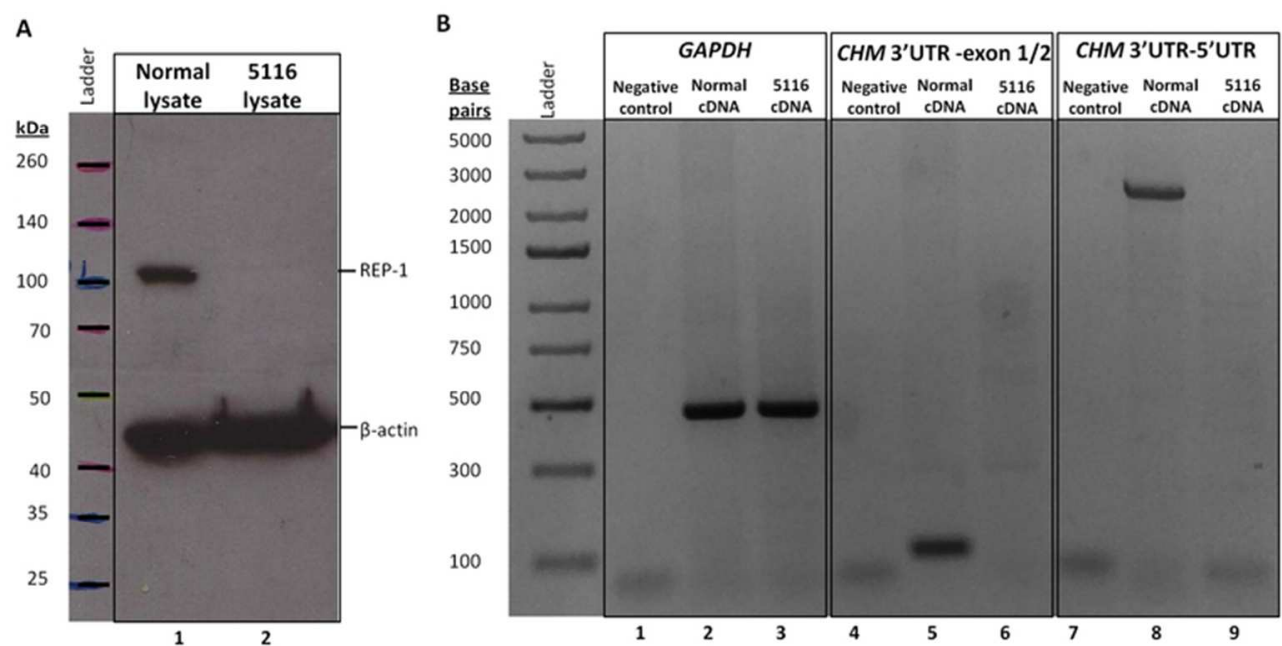

Figure 4. Molecular confirmation of choroideremia in patient 5116.

(A) Patient 5116 lacks REP-1. Western blot results show the absence of a $\sim 100 \mathrm{kDa}$ band corresponding to REP-1 in a lymphoblastoid cell line derived from the patient (lane 2), which is present in a normal control (lane 1). A $\beta$-actin antibody was used as a loading control to ensure an adequate protein sample in each lane, with the $42 \mathrm{kDa}$ band present in both samples.

(B) Patient 5116 lacks CHM mRNA. CDNA synthesized from the mRNA harvested from a patient generated lymphoblastoid cell line was used as template for PCR. Lanes 2 and 3 show a 475 bp band resulting from the amplification of the GAPDH control housekeeping gene from 5116 and a normal control, indicating cDNA of adequate quality. Lanes 6 and 9 demonstrate an absence of amplification from the patient's cDNA of both partial and full length coding sequence, respectively, as compared to PCR products sized 93 and 2200 base pairs amplified from normal cDNA observed in lanes 5 and 10.

Figure 4

$32 \times 16 \mathrm{~mm}(600 \times 600 \mathrm{DPI})$ 


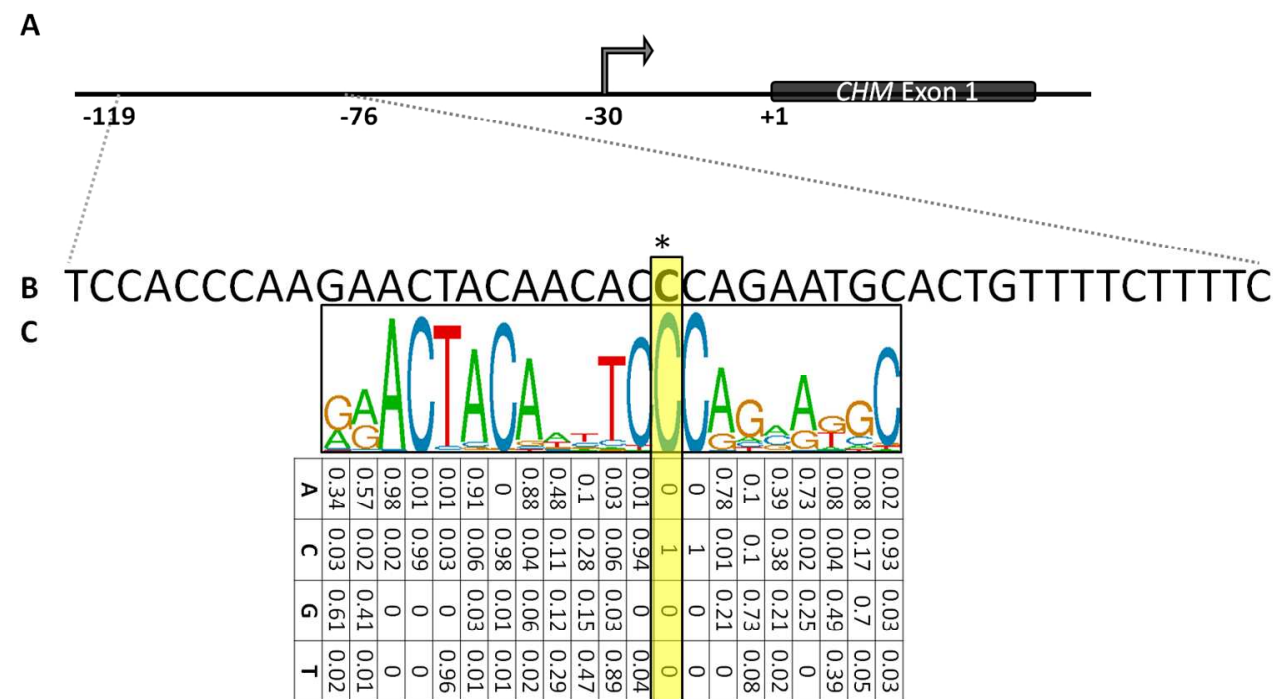

Figure 5. Consensus binding sequences for transcription factor ZNF143. (A) Partial map of the human CHM gene; arrow indicates transcription start site. (B) Expanded sequence of the minimal CHM promoter from C.-119 to C.-76, as identified through the analysis of progressive deletion constructs. Position c.-98 is marked with an asterisk. (C) Sequence logo derived from publically available ChIP-seq data released as part of the ENCODE project, with the position weighted matrix below (ENCODE Project Consortium, 2012). An invariant C is found at position corresponding to c.-98 of CHM.

\section{Figure 5}

$439 \times 257 \mathrm{~mm}(96 \times 96 \mathrm{DPI})$ 


\begin{tabular}{|c|c|c|c|c|c|c|c|c|c|c|}
\hline Patient & $\begin{array}{l}\text { Age at } \\
\text { exam }\end{array}$ & Sex & Genotype & $\mathrm{OD} / \mathrm{OS}$ & BCVA & $\begin{array}{c}\text { ERG } \\
\text { Scotopic } \\
\text { b-wave, } \mu \mathrm{V}\end{array}$ & $\begin{array}{c}\text { ERG } \\
\text { Photopic } \\
\text { b-wave, } \mu \mathrm{V}\end{array}$ & $\begin{array}{c}\text { ERG } \\
30-\mathrm{Hz} \\
\text { flicker, } \mu \mathrm{V}\end{array}$ & Visual Field & Fundus \\
\hline \multirow{2}{*}{5116} & \multirow{2}{*}{76} & \multirow{2}{*}{ M } & \multirow{2}{*}{ c. $-98 \mathrm{C}>\mathrm{A}$} & OD & $6 / 19$ & 0 & 0 & 0.2 & $<5$ degrees & $\begin{array}{l}\text { Widespread chorioretinal } \\
\text { atrophy }\end{array}$ \\
\hline & & & & OS & $6 / 15$ & 0 & 0 & 0.2 & $<5$ degrees & $\begin{array}{l}\text { Widespread chorioretinal } \\
\text { atrophy }\end{array}$ \\
\hline 5149 & - & M & c. $-98 \mathrm{C}>\mathrm{A}$ & \multicolumn{7}{|c|}{ Affected; not examined } \\
\hline \multirow{2}{*}{5113} & \multirow{2}{*}{33} & \multirow{2}{*}{$\mathrm{F}$} & \multirow{2}{*}{$\begin{array}{c}\text { c.- } 98 \mathrm{C} / \mathrm{A} \\
\text { heterozygous }\end{array}$} & OD & $6 / 6$ & \multicolumn{4}{|c|}{ Not tested } & Carrier signs \\
\hline & & & & OS & $6 / 6$ & $\downarrow 50 \%$ & Normal & $\downarrow 50 \%$ & Not tested & Carrier signs \\
\hline 5147 & & M & c. $-98 \mathrm{C}$ & \multicolumn{7}{|c|}{ Unaffected; not examined } \\
\hline \multirow{2}{*}{111} & \multirow{2}{*}{35} & \multirow{2}{*}{ M } & \multirow{2}{*}{ c. $-98 \mathrm{C}>\mathrm{T}$} & OD & $6 / 5$ & \multicolumn{4}{|c|}{ Not tested } & $\begin{array}{l}\text { Focal areas of chorioretinal } \\
\text { atrophy in periphery }\end{array}$ \\
\hline & & & & OS & $6 / 5$ & \multicolumn{4}{|c|}{ Not tested } & $\begin{array}{c}\text { Focal areas of chorioretinal } \\
\text { atrophy in periphery }\end{array}$ \\
\hline 151 & - & M & c. $-98 \mathrm{C}>\mathrm{T}$ & \multicolumn{6}{|c|}{ Data unavailable } & Chorioretinal atrophy \\
\hline \multirow[t]{2}{*}{161} & \multirow[t]{2}{*}{37} & \multirow[t]{2}{*}{ M } & \multirow[t]{2}{*}{ c. $-98 \mathrm{C}>\mathrm{T}$} & OD & $\begin{array}{c}\text { Count } \\
\text { fingers } \\
\text { vision } \\
\end{array}$ & \multicolumn{3}{|c|}{ Not tested } & $\begin{array}{l}\text { Moderately } \\
\text { constricted }\end{array}$ & $\begin{array}{l}\text { Small central areas of } \\
\text { functional retina }\end{array}$ \\
\hline & & & & OS & $6 / 36$ & \multicolumn{3}{|c|}{ Not tested } & $\begin{array}{l}\text { Moderately } \\
\text { constricted }\end{array}$ & $\begin{array}{l}\text { Small central areas of } \\
\text { functional retina }\end{array}$ \\
\hline 190 & - & M & c. $-98 \mathrm{C}$ & \multicolumn{7}{|c|}{ Unaffected; not examined } \\
\hline 192 & - & $\mathrm{F}$ & $\begin{array}{c}\text { c. }-98 \mathrm{C} / \mathrm{T} \\
\text { heterozygous }\end{array}$ & \multicolumn{7}{|c|}{ Not examined } \\
\hline
\end{tabular}


3 Homo sapiens (human)

5 Pan troglodytes (chimp)

Pongo abelii (orangutan)

Callithrix jacchus (marmoset)

Mus musculus (mouse)

10 Rattus norvegicus (rat)

11 Equus caballus (horse)

12 Canis familiaris (dog)

14 Pteropus vampyrus (megabat)

15 Dasypus novemcinctus (armadillo)

16 Monodelphis domestica (opossum)

18 Anolis carolinensis (lizard)

19 Gallus gallus (chicken)

20 Xenopus tropicalis (X. tropicalis)

${ }_{2}{ }_{2}$ ani rerio (zebrafish)

\section{3}

25

26

26
27
28

29

30

32

34

35

36

37

38

39

40

41

42

43

44

45

46

47

48

Base c.-98 and its corresponding location in other species is highlighted

tcca--cccaa-gaactacaa-cac tcca--cccaa-gaactacaa-cac ttccatcccaa-gaactacaa-cac tcca--cccaa-gaactacaa-cac gcgt--ctcta-gaactacaa-cac gcta--cccta-aaactacaa-cac gcca--cccaa-gaactacaa-tac gcca--cccac-gaactacaa-tat gcca--cccaa-ggactacaa-tac tcaa--cca-atgaactacaa-tac tcct--gccac-gaactacaa-atc $--------a-g a a c t a c a g-c a a$

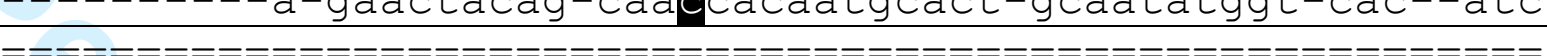

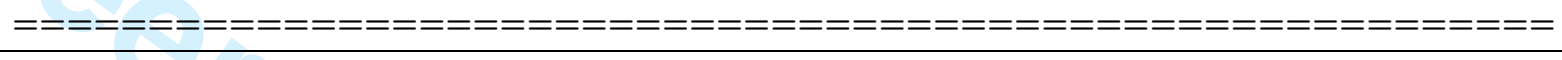

cagaatgcact-g-----tttt-ctt--ttc cagaatgcact-g-----tttt-cct--ttc cagaatgcatt-g-----tttttcct--ttc cagaatgcatt-g-----tttt-cct--ttc cagaatgcact-g-----tttt-tcc--ttc cagaatgcact-g-----tttt-tcc--ttc cagaatgcact-g-----tttt-ccc--ttc cagaatgcatt-g-----tttt-tcc--ttc cagaatgcact---------tt-tcc----c cagaatgcact-a-----tttt-cct--ttc cagaatgcatt-g-----cgct-ccc----g cacatgcact-gcaatatggt-cac--atc 